\section{INGENIERÍA SÍSMICA}

Revista de Ingeniería Sísmica

ISSN: 0185-092X

javiles@tlaloc.imta.mx

Sociedad Mexicana de Ingeniería Sísmica

México

Rodríguez, Mario E.; Restrepo, José I.

PRÁCTICA Y DISEÑO SÍSMICO DE EDIFICIOS EN MÉXICO - CAMBIOS NECESARIOS

Revista de Ingeniería Sísmica, núm. 86, enero-junio, 2012, pp. 89-118

Sociedad Mexicana de Ingeniería Sísmica

Distrito Federal, México

Disponible en: http://www.redalyc.org/articulo.oa?id=61824407004

- Cómo citar el artículo

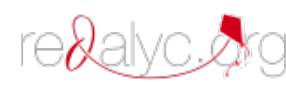

- Número completo

- Más información del artículo

Página de la revista en redalyc.org

Sistema de Información Científica

Red de Revistas Científicas de América Latina, el Caribe, España y Portugal Proyecto académico sin fines de lucro, desarrollado bajo la iniciativa de acceso abierto 


\title{
PRÁCTICA Y DISEÑO SÍSMICO DE EDIFICIOS EN MÉXICO - CAMBIOS NECESARIOS
}

\author{
Mario E. Rodríguez ${ }^{(1)}$ y José I. Restrepo ${ }^{(2)}$
}

\begin{abstract}
RESUMEN
En este trabajo se hace una revisión crítica al Reglamento de Construcciones del Distrito Federal del 2004 (RCDF) en su parte de diseño sísmico, y se proponen algunos cambios en los requisitos de diseño sísmico para edificios, así como procedimientos congruentes con investigaciones recientes, con el propósito de mejorarlo. Se propone mejorar la definición de espectros de diseño, así como cambios en su empleo para lograr diseños más transparentes y racionales. Se demuestra la necesidad de cambios en la normativa actual para evitar el empleo de estructuras con insuficiente capacidad de deformación lateral, también para que en el análisis de estructuras se evite sobrestimar la rigidez lateral de éstas, así como para mejorar los procedimientos de diseño sísmico de apéndices y diafragmas en edificios.
\end{abstract}

Palabras clave: diseño sismorresistente, espectros de diseño, conexiones en prefabricados, rigidez lateral, aceleraciones horizontales de piso, diafragmas

\begin{abstract}
Seismic design provisions given by the 2004 Mexico City Building Code (MCBC) are reviewed in this paper. To improve these provisions, and based on recent research findings, changes for some of these provisions are proposed. The paper proposes improving the definition of current seismic design spectra, to lead to rational and transparent seismic design procedures. It is shown that changes are needed in the MCBC procedures for prohibiting the use of structures with insufficient lateral deformation capacity, and for appropriate evaluation of lateral stiffness of structures in order to avoid the overestimation of this stiffness, as well as for better seismic design procedures of diaphragms and appendixes.
\end{abstract}

Keywords: earthquake resistant design, design spectra, connections in precast structures, lateral stiffness, horizontal floor accelerations, diaphragms.

\section{INTRODUCCIÓN}

Los propietarios de diversas obras civiles que se construyen aceptan de manera implícita que cumpliendo la normativa de construcción existente, estas obras tendrían una vida útil de 50, 100 o más años, periodo en el cual incluso no esperan daños importantes por sismo en estas obras. Eso no es parte de los objetivos de los reglamentos de construcción en zonas sísmicas. El desengaño de los propietarios y/o usuarios es evidente después de sismos, incluso moderados. El problema se agrava porque muchos ingenieros que ejercen la práctica de diseño sísmico en México y que siguen por ejemplo los requisitos del

1. Instituto de Ingeniería, Universidad Nacional Autónoma de México, Apartado Postal 70-290, Ciudad

Universitaria, CP 04510, México DF, mrod@ servidor.unam.mx

2. University of California at San Diego, San Diego, California, USA, jrestrepo@ soe.ucsd.edu 
RCDF, dan por hecho que estos requisitos llevan a diseños óptimos de edificios (en costos y seguridad), más aun si en los procedimientos de análisis y diseño emplean programas de cómputo elaborados. En este trabajo se demuestra que aún así, no se estaría llegando a diseños óptimos o garantizando un diseño sísmico adecuado.

Es conocida la influencia del RCDF en otras normativas existentes en el país, por lo que las mejoras que se logren en la primera serán benéficas para las segundas. Este trabajo pone en evidencia aspectos de la normativa actual de diseño sísmico del RCDF que deberían revisarse, y se proponen posibles cambios. En lo que sigue, la evaluación de la normativa que se lleva a cabo en este trabajo se divide en temas específicos.

\section{LA DEFINICIÓN DE LOS ESPECTROS DE DISEÑO POR SISMO PARA EDIFICIOS EN LA NORMATIVA POR SISMO PARA EL DISTRITO FEDERAL}

El cuerpo principal de las Normas Técnicas Complementarias por Sismo (NTCS) estipula espectros de diseño sísmico en los cuales ya se ha considerado de manera implícita el efecto de la sobrerresistencia existente en estructuras. Es decir, los espectros de diseño estipulados en estas normas en su cuerpo principal ya están reducidos por el factor correspondiente al efecto de sobrerresistencia, factor que en el Apéndice A de las NTCS se denomina $R$ y el cual se emplea de manera explícita en este Apéndice. Se debe mencionar que esta práctica de estipular espectros de diseño reducidos de manera implícita por el factor de sobrerresistencia la emplea también la versión anterior del Manual de Diseño por Sismo de la Comisión Federal de Electricidad (CFE, 1993) y también se emplea en reglamentos específicos para zonas sísmicas fuera del DF, como por ejemplo los reglamentos para el estado de Guerrero, o en otros estados como Jalisco, Michoacán, Colima ú otros. Sin embargo, el Manual de Diseño de Obras Civiles de la CFE (CFE, 2008) sigue un criterio parecido al del Apéndice A de las NTCS, ya que emplea el factor $R$ de manera explícita, así como un factor de redundancia. El factor $R$, así como diversos factores relevantes que intervienen en la definición de las fuerzas sísmicas de diseño se describen en lo que sigue.

La Fig. 1 muestra resultados de la respuesta global de una estructura, en función del cortante basal, $V_{b}$, y del desplazamiento global de la estructura, en este caso el desplazamiento del nivel azotea, $\delta$. La Fig. 1 muestra el cortante basal requerido para que la estructura permanezca elástica para el sismo de diseño, $V_{e}$, el cual se puede expresar sin dimensiones en la forma del llamado coeficiente sísmico, $C_{e}$, el que se define como:

$C_{e}=\frac{V_{e}}{W}$

donde $W$ es el peso de la estructura. La filosofía de los reglamentos es la de diseñar una estructura para que tenga una resistencia menor que $V_{e}$, basado en reconocer su capacidad de comportamiento inelástico. Esta estructura tiene un desplazamiento de fluencia $\delta_{y}$ y un desplazamiento máximo $\delta_{\max }$, Fig. 1 , y su resistencia es la de fluencia, $V_{y}$, la cual en función del coeficiente sísmico $C_{y}$ se expresa como:

$C_{y}=\frac{V_{y}}{W}$

En realidad el cálculo de $V_{y}$ implica análisis del tipo no lineal, por lo que de manera típica los procedimientos de reglamentos de diseño por sismo, basados en herramientas de comportamiento elástico, especifican una resistencia elástica, $V_{s}$. Este cortante en función del coeficiente sísmico $C_{s}$, Fig. 1, se expresa como:

$C_{s}=\frac{V_{s}}{W}$ 
El factor de reducción de fuerzas elásticas, $R_{T}$, se define como:

$$
R_{T}=\frac{C_{e}}{C_{s}}
$$

Reconociendo la ductilidad de la estructura y su capacidad de disipar energía en su comportamiento inelástico, se define el factor de reducción por ductilidad $R_{\mu}$ como:

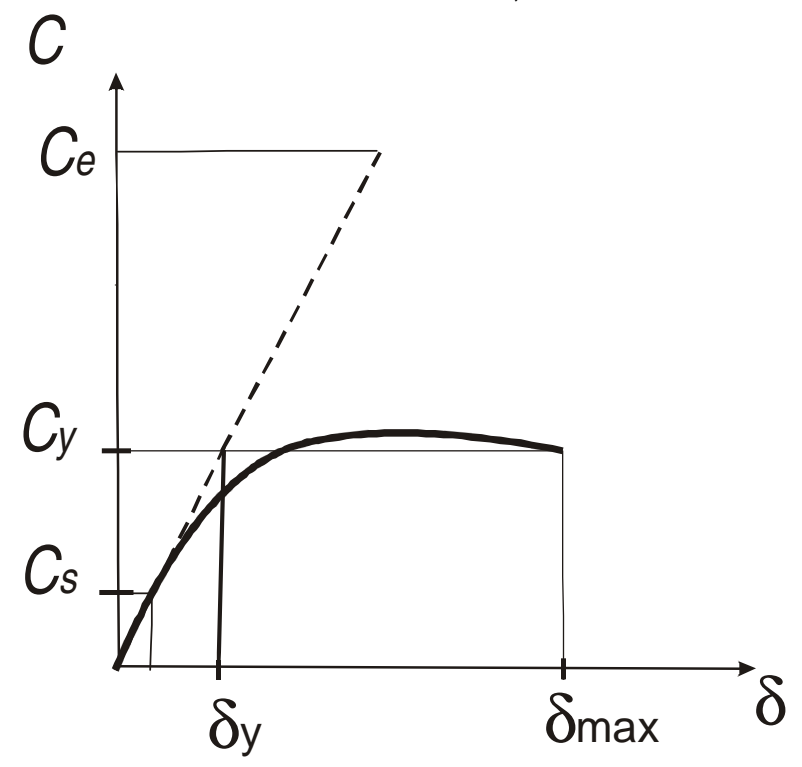

Figura 1. Relación coeficiente sísmico basal-desplazamiento lateral de la estructura

$R_{\mu}=\frac{C_{e}}{C_{y}}$

El factor de sobrerresistencia $R$ se define como:

$$
R=\frac{C_{y}}{C_{s}}
$$

Combinando las ecs (4) a la (6) se obtiene:

$$
R_{T}=R_{\mu} R
$$

De la (4) y (7)

$C_{s}=\frac{C_{e}}{R} \frac{1}{R_{\mu}}$

Las ordenadas de los espectros de diseño "elásticos", especificados por el cuerpo principal de las NTCS, en realidad no corresponden a espectros de respuesta elásticos, ya que por estar divididas por el factor R corresponden al cociente $C_{e} / R$. De acuerdo con esta normativa, estas ordenadas se dividen por el factor de reducción $Q$ ' para obtener la resistencia de diseño $C_{s}$, por lo que de acuerdo con la Ec (8), se tendría $R_{\mu}=Q^{\prime}$. Se debe mencionar que en el Apéndice A de las NTCS se emplea de manera explícita el 
producto $R Q$ ' para reducir el coeficiente sísmico elástico $C_{e}$. Este requisito del cuerpo principal de las NTCS de emplear espectros de diseño ya reducidos por el factor de sobrerresistencia es diferente a la práctica de diseño sísmico en otros países y lleva a las siguientes deficiencias en la práctica de diseño sísmico.

La primera deficiencia es que este criterio de diseño de definición del espectro no lleva a procedimientos de diseño sísmico transparentes y dificulta en la práctica el empleo de conceptos básicos de diseño sísmico. Esto se debe a que las fuerzas "elásticas" se valúan con las ordenadas espectrales reducidas, $C_{e} / R$, y por tanto en realidad son menores que las fuerzas elásticas que corresponderían a las ordenadas espectrales elásticas del sismo de diseño (correspondientes al valor $C_{e}$ ). Además, en algunos casos, como se demuestra en este trabajo, para definir fuerzas sísmicas necesarias para el diseño de diafragmas ó apéndices, es necesario conocer la resistencia $C_{y}$, la que de acuerdo con la Ec (6) se obtiene como el producto $R C_{s}$, lo que indica la necesidad de conocer de manera explícita el valor de $R$ que se emplea en los espectros de diseño.

La segunda deficiencia es que en el cálculo de la demanda de desplazamientos de la estructura de acuerdo con el procedimiento del cuerpo principal de las NTCS, en general el ingeniero estaría calculando desplazamientos bastante menores que los que se tendrían para el sismo de diseño. Estos aspectos se evalúan con detalle en lo que sigue.

Para ilustrar los efectos de considerar de manera implícita el factor $R$ en los espectros de diseño por sismo se muestra los resultados de la Fig. 2. Esta figura muestra ordenadas del espectro de aceleraciones, $a$, expresadas como fracción de la gravedad (se está empleando la notación de las NTCS), $g$, en función del periodo de la estructura $T$, además en esta figura $a_{o}$ es el valor de la ordenada del espectro de aceleraciones correspondiente a $T=0$. La curva identificada como A en la Fig. 2a representa de manera esquemática al espectro de aceleraciones "elástico" de diseño especificado por las NTCS, y corresponden a valores de ordenadas espectrales iguales a $C_{e} / R$. La curva A en la Fig. 2b representa al espectro elástico de diseño de desplazamientos, $S_{d}$, de esta normativa, y sus ordenadas se calculan a partir de los valores de las ordenadas espectrales de la curva A en la Fig. 2a con la siguiente expresión:

$S_{d}=\left(\frac{T}{2 \pi}\right)^{2} a g$

Si el efecto de la sobrerresistencia se tomara en cuenta de manera explícita, los espectros de aceleraciones y de desplazamientos correspondientes serían los mostrados en las curvas B de las Figs 2a, y $2 \mathrm{~b}$, respectivamente, y corresponden a las ordenadas de aceleraciones y desplazamientos de las curvas $\mathrm{A}$ multiplicadas por el factor $R$.

La inspección de la Fig. 2 ilustra de manera gráfica los dos deficiencias anteriormente mencionadas de la normativa actual. La primera, que las fuerzas sísmicas elásticas especificadas por las NTCS para el sismo de diseño, cuyos valores están asociados a la curva A en la Fig. 2a, en realidad son menores que las que corresponderían a las fuerzas elásticas para este sismo, cuyos valores corresponden a la curva B de la Fig. 2a. La segunda deficiencia se infiere de la inspección de la Fig. 2b. En ella se aprecia que los desplazamientos que corresponderían al sismo de diseño, curva B en la Fig. 2b, son mayores que los resultantes de emplear los espectros de diseño del cuerpo principal de las NTCS, curva A de la Fig. 2 b. Además, se debe observar que de acuerdo con las NTCS, la Fig. 2a muestra que la aceleración máxima del terreno en las curvas A y B son diferentes, error que se debe corregir porque éste es un parámetro 
independiente de la respuesta estructural. Como se demuestra posteriormente, se requiere el valor de este parámetro para valuar las fuerzas inerciales que actúan en apéndices y/o en el plano de sistemas de piso.
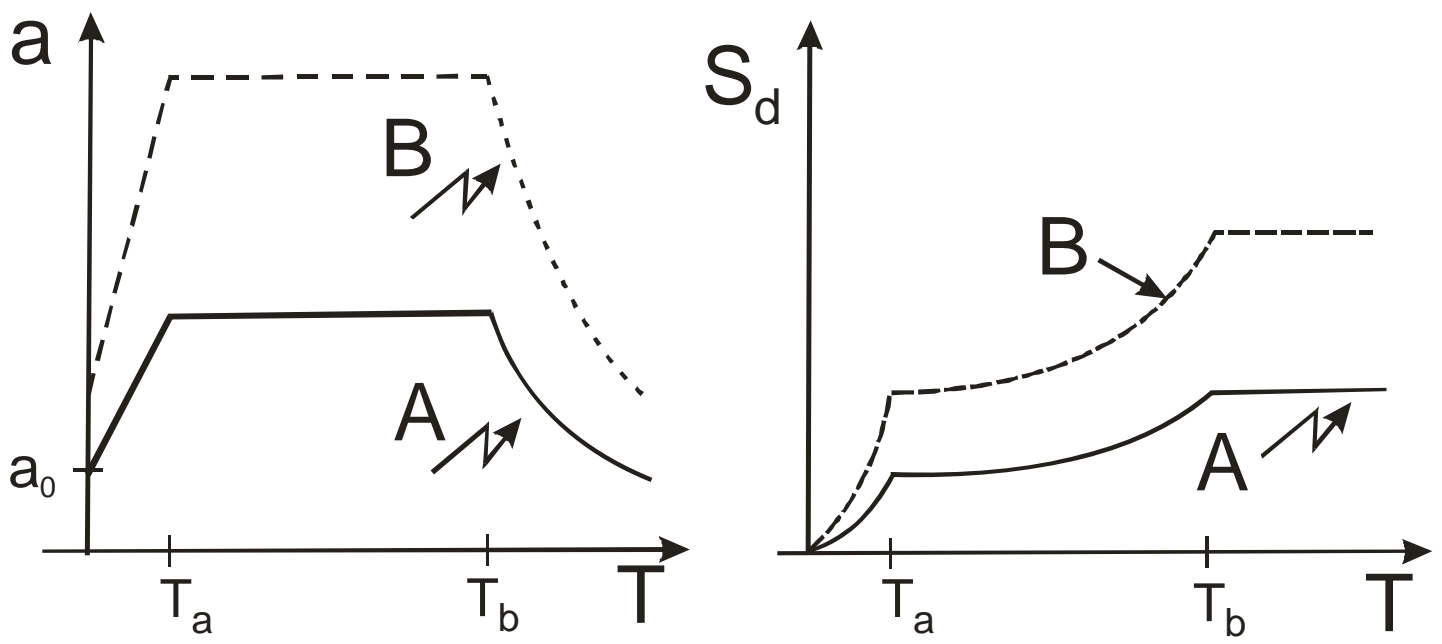

Figura 2. Espectros de diseño de las NTCS (curva A) y con sobrerresistencia (curva B)

Una evaluación del factor de sobrerresistencia, $R$, indica que depende de varios factores, como tipo de estructura, materiales, numero de niveles, entre otros. En la literatura se menciona valores para este parámetro entre 2 y 3, e incluso mayores si se considera las propiedades esperadas de los materiales en lugar de las especificadas (Uang, 1991). Resultados de ensayes en México de un edificio de dos niveles de concreto reforzado prefabricado, a escala un medio, al cual se aplicó en laboratorio cargas laterales cíclicas reversibles (Rodríguez y Blandón, 2005), muestran para $R$ un valor igual a 2.6, como se aprecia en la Fig. 3. En esta figura, el cortante basal aplicado, $V_{b}$, en la estructura se expresa sin dimensiones dividiendo $V_{b}$ entre la resistencia correspondiente a la aparición de la primera articulación plástica, calculada con los requisitos del RCDF, $V_{b D F}$. El eje de las abscisas muestra la distorsión global, $D_{r}$, parámetro que se define como el cociente entre el desplazamiento de la azotea, $\delta$, y la altura del edificio, $H$. Se debe mencionar que los valores que aquí se comentan para $R$ en general son mayores que los especificados por el Apéndice A de las NTCS, cuyos valores se determinaron de manera empírica (Ordaz et al., 2000). Este Apéndice especifica para $R$ valores entre 2 y 2.5 para estructuras con periodo fundamental menor que $T_{a}$, y para estructuras con periodo fundamental mayor que $T_{a}, R$ se especifica igual a 2, donde $T_{a}$ es el periodo característico de los espectros de diseño.

Si con base en esta información consideramos que el factor $R$ en general es mayor que dos, la probable demanda de desplazamientos laterales en estructuras sería mayor, en al menos un 100\%, que la demanda de desplazamientos laterales calculada de acuerdo con el cuerpo principal de las NTCS. La normativa actual de diseño sísmico, tanto para el DF, como para otros lugares del país, intenta corregir esta deficiencia en opinión de los autores sin éxito. Esto se debe a que los valores de la demanda de desplazamientos laterales que calcula el ingeniero empleando el procedimiento del cuerpo principal de las NTCS, los compara con límites ficticios de desplazamientos laterales, en realidad menores que los asociados a las posibles capacidades de distorsión. Esto ha llevado a confusiones serias en la práctica de diseño sísmico de estructuras en nuestro medio, así como en la interpretación de su comportamiento sísmico. Por ejemplo, la resistencia $C_{s}$, valuada con las fuerzas sísmicas elásticas especificadas por el cuerpo principal de las NTCS y reducidas por el factor $Q$, no sería directamente comparables con resultados medidos en ensayes en laboratorio de la resistencia lateral de una estructura diseñada de 
acuerdo con las NTCS, ya que a esta última le correspondería la resistencia $C_{y}$, la que de acuerdo con la Ec (6) estaría amplificada por el factor $R$ respecto a las primeras. Además, las distorsiones medidas en estos ensayes tampoco podrían ser directamente comparables con las distorsiones límites especificadas por las NTCS, y serían bastante menores que estas últimas. Lo mismo ocurriría en ensayes de elementos arquitectónicos de fachada o de elementos "no estructurales", donde es relevante la información de su capacidad de deformación lateral.

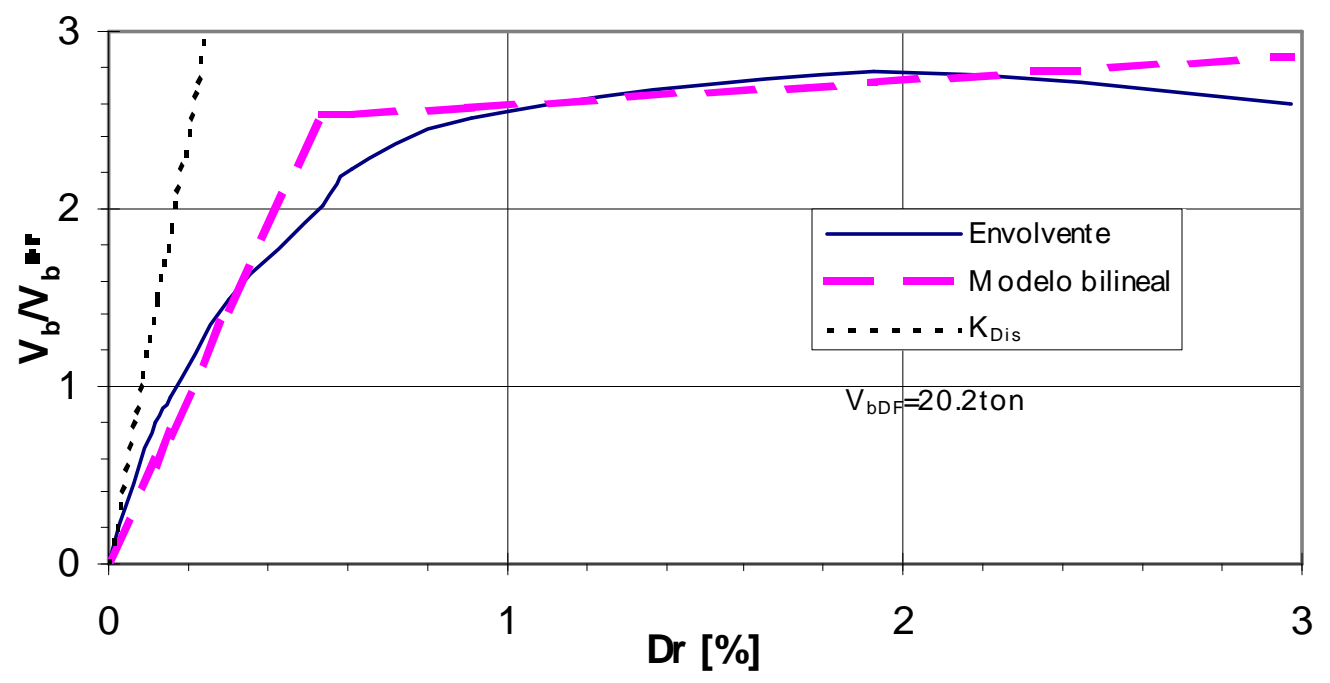

Figura 3. Relación cortante basal adimensional versus distorsión global en una estructura de concreto reforzado de dos niveles. Envolvente de resultados experimentales y modelo bilineal (Rodríguez y

Blandón, 2005)

Con el objeto de ilustrar de manera cuantitativa algunas de las observaciones anteriores, en lo que sigue se analizan resultados de la evaluación de la respuesta estructural de un edificio diseñado de acuerdo con el RCDF, cuando éste se somete a un registro de aceleraciones típico de la zona de más alta intensidad sísmica del DF.

Ejemplo de un edificio de concreto reforzado de 12 niveles diseñado de acuerdo con el Reglamento de Construcciones del DF

El edificio analizado se ubica sobre suelo tipo IIIc en el DF y tiene un sistema estructural a base de marcos de concreto reforzado, de doce niveles. La Fig. 4 muestra la configuración en planta y en elevación del edificio. Este se analizó y diseñó con base en las NTCS y las Normas Técnicas Complementarias para Diseño y Construcción de Estructuras de Concreto (NTCC, 2004), considerando la distorsión máxima de entrepiso, $d_{r D I S}$, igual a 0.012. El coeficiente sísmico de diseño elástico en la zona plana del espectro para esta zona del DF es 0.4. Se empleó un factor de reducción, $Q$ ', igual a 4, y se consideró que el uso de los edificios sería de oficinas. Para el diseño de los elementos estructurales se consideró una resistencia especificada a la compresión del concreto, $f_{c}$, igual a $34.3 \mathrm{MPa}\left(350 \mathrm{~kg} / \mathrm{cm}^{2}\right)$ y una resistencia a la fluencia del acero de refuerzo, $f_{y}$, de $411.6 \mathrm{MPa}\left(4200 \mathrm{~kg} / \mathrm{cm}^{2}\right)$. Para las cargas gravitacionales se emplearon los siguientes valores sin factores de carga:

Elementos no estructurales

Firme $e=6 \mathrm{~cm}$
$216 \mathrm{Kg} / \mathrm{m}^{2}$

$144 \mathrm{Kg} / \mathrm{m}^{2}$ 
Carga adicional

Vigas

Carga Muerta

Carga viva
$40 \mathrm{Kg} / \mathrm{m}^{2}$

$220 \mathrm{Kg} / \mathrm{m}^{2}$

$620 \mathrm{Kg} / \mathrm{m}^{2}$

$180 \mathrm{Kg} / \mathrm{m}^{2}$
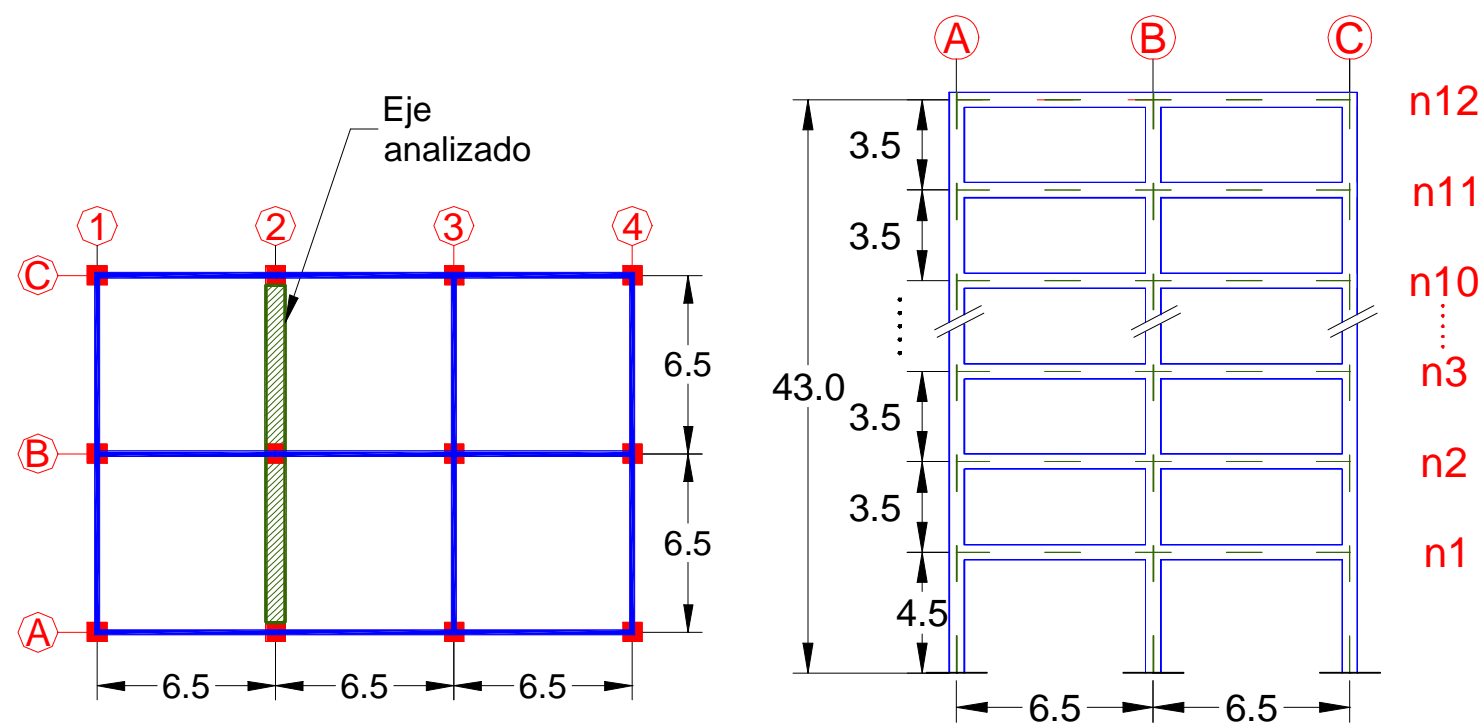
a) Planta
b)Elevación

Figura 4 Planta y elevaciones típicas del edificio que se estudia (dimensiones en metros)

La Tabla 1 muestra valores típicos empleados para el diseño, tales como las dimensiones de secciones transversales de vigas y columnas, el peso total del edificio, $W$, y el coeficiente sísmico de diseño reducido, $c_{D I S}$. Esta tabla también muestra el valor de la distorsión calculada máxima de entrepiso, $d_{r D I S}$, y del periodo fundamental de vibración del edificio, $T_{D I S}$. Estos valores fueron obtenidos analizando el edificio con el programa de cómputo ETABS (CSI, 2008), aplicando los requisitos especificados por el $\mathrm{RCDF}$, considerando para las vigas una sección del tipo rectangular y una inercia igual a la mitad de la inercia bruta, y para las columnas la inercia bruta, así como nudos rígidos para las vigas. Para el diseño sísmico de este edificio se empleó el análisis modal espectral. Sus resultados dieron una fuerza cortante basal máxima, $V_{b}$, igual a $0.08 \mathrm{~W}$, el cual corresponde también al valor mínimo que se permite con un análisis modal espectral, es decir el $80 \%$ del valor de cortante basal que se emplearía en un análisis estático. La Tabla 2 muestra detalles del refuerzo longitudinal de vigas y columnas del edificio. La Fig. 5 muestra armados de secciones típicas de vigas y columnas del marco analizado. 
Tabla 1. Características de diseño con $Q=4$ para el edificio en el DF

\begin{tabular}{|l|l|}
\hline Nombre & $\mathrm{DF} 12 \mathrm{n}-12$ \\
\hline$W$ (ton) & 2,436 \\
\hline$c_{D I S}$ & 0.1 \\
\hline$T_{D I S}(\mathrm{seg})$ & 1.69 \\
\hline$d_{r \text { DIS }}$ & 0.012 \\
\hline Sección vigas (metros) & $0.30 \mathrm{x} 0.70$ \\
\hline Sección Columnas (metros) & $0.80 \times 0.80$ \\
\hline
\end{tabular}

Tabla 2. Refuerzo longitudinal en vigas y columnas del edificio

\begin{tabular}{|c|c|c|c|c|}
\hline \multicolumn{2}{|c|}{ VIGAS } & \multicolumn{3}{|c|}{ COLUMNAS } \\
\hline NIVEL & $\begin{array}{c}\text { REFUERZO LECHO } \\
\text { SUPERIOR }\end{array}$ & NIVEL & REFUERZO & CUANTÍA (\%) \\
\hline $1-8$ & $4 \phi 1^{\prime \prime}$ & $1-3$ & $24 \phi 1-1 / 4{ }^{\prime \prime}$ & 2.97 \\
\hline $9-12$ & $3 \phi 1^{\prime \prime}$ & $4-8$ & $16 \phi 1-1 / 4{ }^{\prime}$ & 1.98 \\
\hline & & $9-12$ & $4 \phi 11 / 4{ }^{\prime}+8 \phi 1^{\prime}$, & 1.13 \\
\hline
\end{tabular}

Los refuerzos de lecho superior e inferior son iguales

El análisis dinámico no lineal para el marco se efectuó con el programa Ruaumoko (Carr, 2002), el cual permite valuar la respuesta dinámica no lineal de estructuras. Es conocido que para definir las fuerzas de amortiguamiento en la estructura se supone que estas fuerzas son del tipo viscoso, y por simplicidad la matriz de amortiguamiento de la estructura usualmente se calcula como una combinación de la matriz de rigidez y de masa, procedimiento que lleva al conocido amortiguamiento de Rayleigh. Este procedimiento puede llevar a sobrestimar el efecto de amortiguamiento para los modos superiores (Carr, 2002). Por este motivo, en este análisis se empleó el modelo de amortiguamiento de Caughey (Carr, 2002), con una fracción de amortiguamiento crítico, $\xi$, constante para todos los modos, en vez del procedimiento tradicional mencionado y usado en programas de cómputo comúnmente empleados en la práctica, en el cual se dan valores a $\xi$ sólo para dos modos. El valor de $\xi$ considerado en los análisis fue $5 \%$ para todos los modos, valor empleado para ser congruente con los valores de la fracción de amortiguamiento crítico implícito en los espectros de diseño de las NTCS. Sin embargo, este valor para un análisis dinámico no lineal podría estar sobrestimado, ya que resultados de ensayes en mesa vibradora de un edificio de concreto reforzado de 7 niveles (Panagiotou, 2008; Martinelli y Filippou, 2009) indican que emplear para $\xi$ valores mayores que $2 \%$, cuando se lleven a cabo análisis dinámicos no lineales, llevan a subestimar de manera importante los desplazamientos laterales en la estructura. 


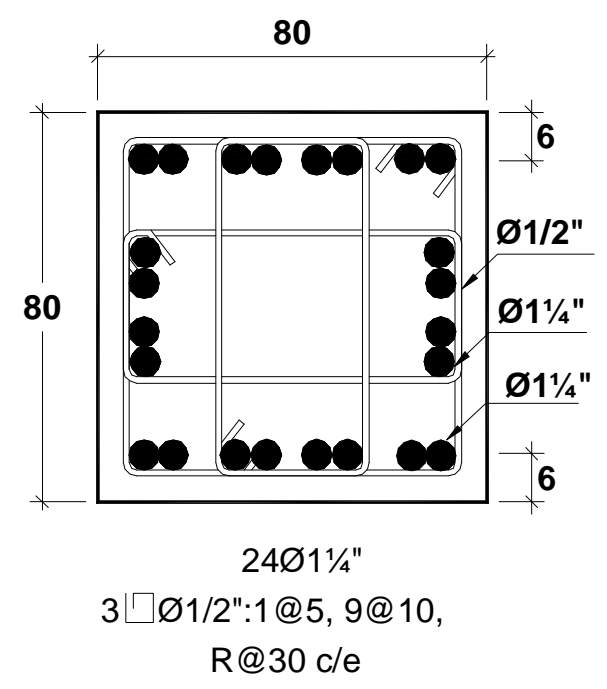

a) Columnas niveles $1-3$

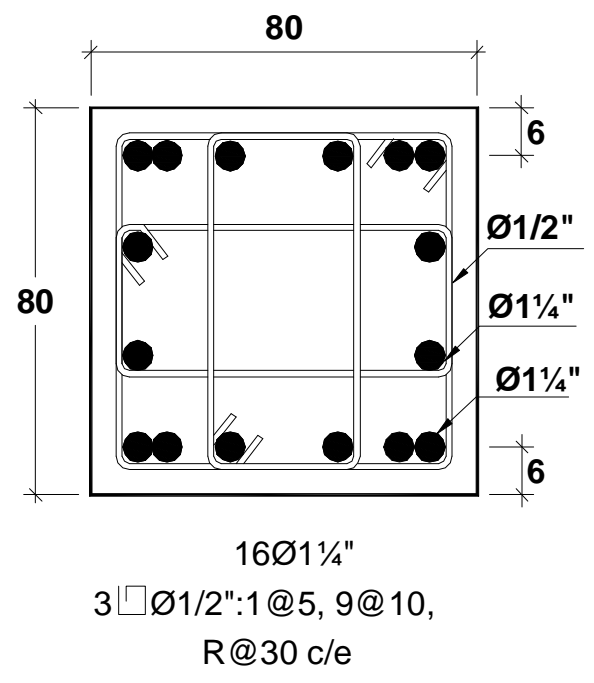

b) Columnas niveles $4-8$

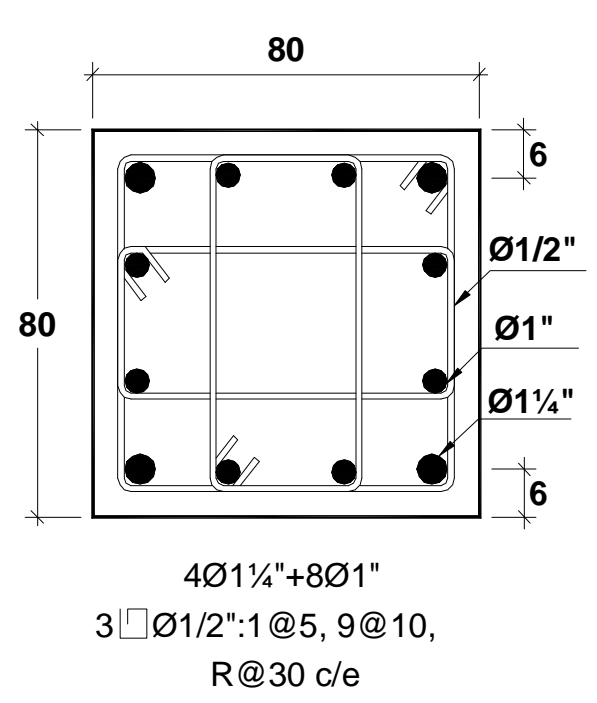

c) Columnas niveles 9-12

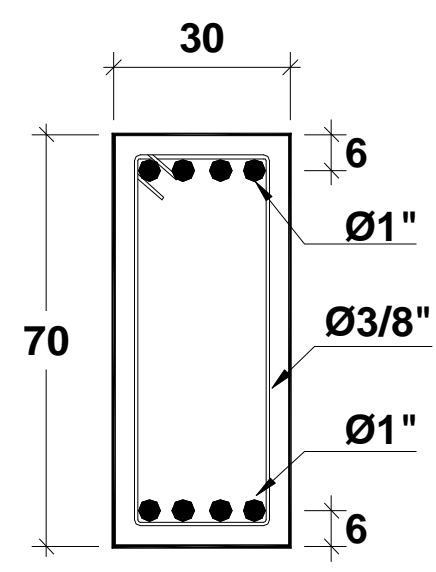

$1 \square$ 1 R@20 c/e

d) Trabes niveles 1-8

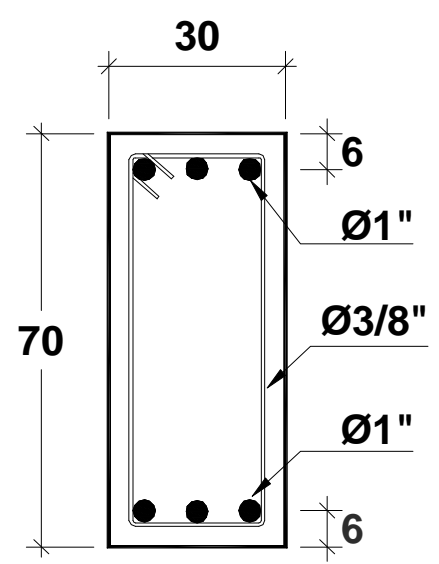

$1 \square \varnothing 3 / 8 ": 1 @ 5,7 @ 10$ R@20 c/e

e) Trabes niveles 9-12

Figura 5. Dimensiones y detalles de refuerzo típicos en columnas y trabes del marco $(\mathrm{cm})$

Se empleó el método de integración de Newmark $(\beta=0.25)$ por ser incondicionalmente estable, con un paso de integración igual a 0.001. Se tuvo en cuenta en el análisis el efecto P-Delta (Carr, 2002). Para definir las relaciones momento-curvatura de las secciones críticas de vigas y columnas necesarias para el programa Ruaumoko, se consideró los valores medios de los parámetros característicos que definen la curva-esfuerzo deformación del acero de refuerzo longitudinal, para lo cual se emplearon los valores propuestos por Rodríguez y Botero (1995). Para la curva esfuerzo-deformación del concreto se consideró el efecto del confinamiento considerando el modelo de Park et al. (1982). Para estas relaciones momento- 
curvatura se empleó la regla de histéresis de Takeda modificado (Carr, 2002), en la que la rigidez de descarga, $k_{u}$, se valuó como:

$k_{u}=k_{o} \mu_{\phi}^{-\alpha}$

donde $\mu_{\phi}$ es el factor de ductilidad de curvatura, y $k_{o}$ es la rigidez inicial del sistema. El parámetro de rigidez de descarga, $\alpha$, se tomó igual a 0.5 .

El registro de aceleraciones empleado para el análisis dinámico no lineal fue el conocido registro de la SCT, componente E-W, obtenido en la zona de suelo blando del DF, en el terremoto del 19 de septiembre de 1985. La Tabla 3 muestra algunos resultados obtenidos del análisis paso a paso para el marco en estudio. Esta tabla muestra los valores del peso sísmico total del marco, $W$, así como del periodo fundamental de vibración, $T_{R M K}$, obtenido con el programa Ruaumoko. La Tabla 3 también muestra valores máximos calculados con este programa para el coeficiente sísmico, $c_{M a x}$, así como para la distorsión global máxima, $D_{r \text { Max }}$, y para la distorsión máxima de entrepiso en el marco, $d_{r M a x}$, la cual correspondió al cuarto nivel.

En esta investigación también se obtuvieron valores para los parámetros $M_{v e}, \mathrm{y} M_{v o}$, definidos como el momento de volteo máximo en un edificio que se requiere para lograr respuesta elástica por sismo, y el momento de volteo máximo que se obtiene de un análisis no lineal en el tiempo, respectivamente. El momento de volteo en un edificio, $M_{v}$, se define como la suma de los momentos respecto a la base de las fuerzas inerciales, $F_{i}$, y la contribución del peso por nivel, lo que lleva a:

$M_{V}=\sum_{i=1}^{n}\left[\ddot{U}_{i}(t) \cdot m_{i} \cdot h_{i}+w_{i} \cdot \Delta_{i}(t)\right]$

donde $\ddot{U}_{i}(t)$ es la aceleración horizontal absoluta del piso $i$ con una masa $m_{i}$, ubicada a una altura de desplante $h_{i}$, para el tiempo $t$, y $w_{i}$ es el peso del nivel $i$ que presenta un desplazamiento lateral $\Delta_{i}(t)$.

La Tabla 3 muestra los valores calculados de los momentos máximos de volteo en la base, $M_{v e}$, y $M_{v o}$, los que para expresarlos sin dimensiones se dividieron con el producto $\mathrm{WH}$.

Tabla 3. Resultados del análisis dinámico no lineal del marco

\begin{tabular}{|l|l|}
\hline Marco & 12 Niveles \\
\hline Nombre & DF12n-12 \\
\hline$W$ (ton) & 609 \\
\hline$T_{R M K}($ seg) & 1.73 \\
\hline$c_{\text {Max }}$ & 0.231 \\
\hline$D_{\text {rMax }}$ & 0.0144 \\
\hline$d_{r \text { Max }}$ (nivel 4) & 0.0254 \\
\hline$M_{v e} /(W \cdot H)$ & 0.313 \\
\hline$M_{v o} /(W \cdot H)$ & 0.143 \\
\hline$R_{M}=M_{v e} / M_{v o}$ & 2.19 \\
\hline
\end{tabular}

El valor del parámetro $R_{M}$, factor de reducción de la respuesta elástica, el cual se emplea posteriormente, se define en la Ec (12) y su valor para el caso en estudio se muestra en la Tabla 3, 
$R_{M}=\frac{M_{v e}}{M_{v o}}$

La Fig. 6 muestra valores del coeficiente sísmico $V_{b} / W$ obtenidos con el programa de análisis dinámico no lineal. El valor máximo para este cociente fue 0.231. De acuerdo con la Ec (6), si se considera este valor y la resistencia $C_{s}$ requerida por el análisis modal espectral $(0.08 \mathrm{~W})$, el valor del factor de sobrerresistencia, $R$, es 2.9.

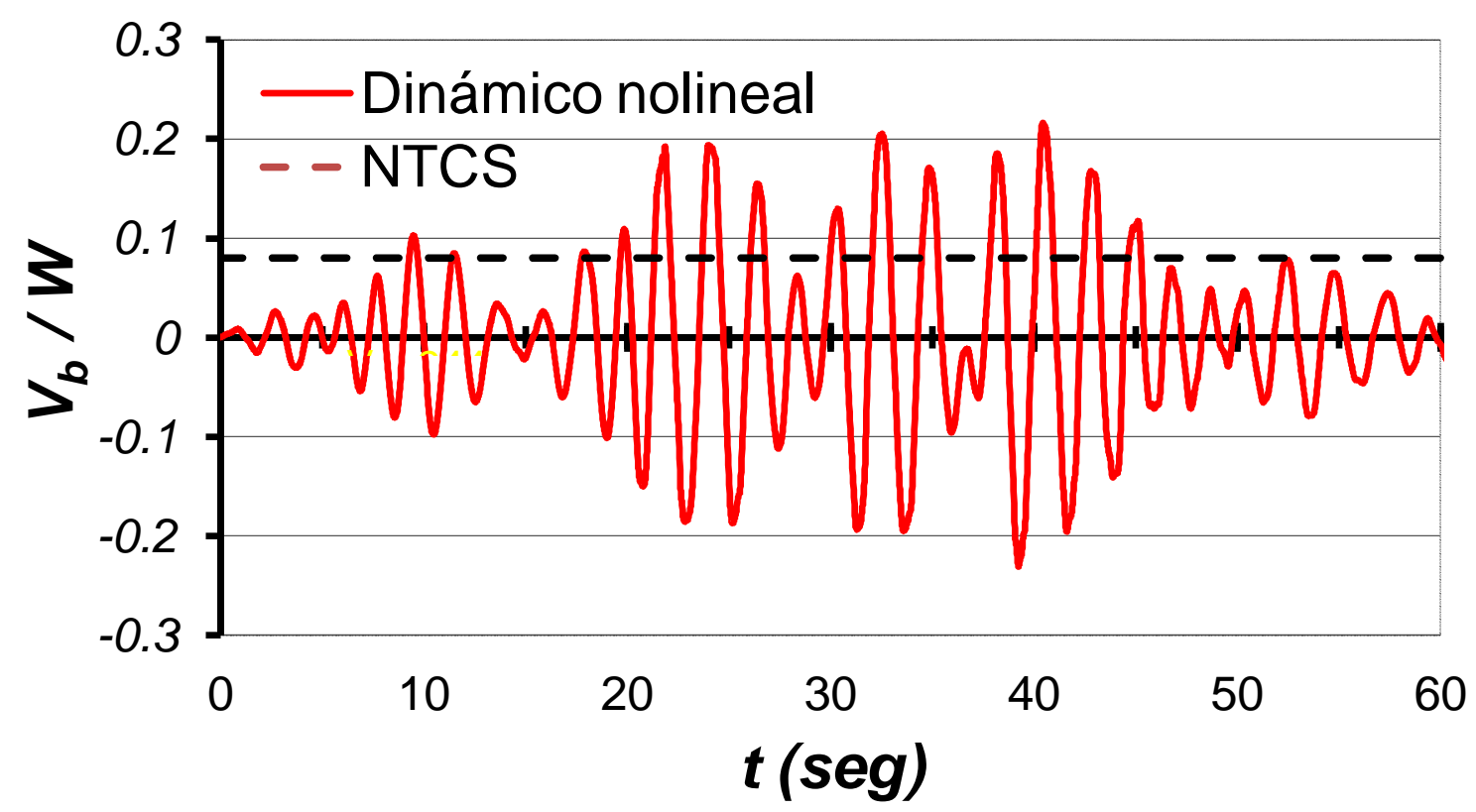

Figura 6. Coeficiente sísmico basal en el tiempo obtenido con el análisis dinámico no lineal y con las NTCS

La Fig. 7 muestra valores calculados de la respuesta dinámica no lineal para el momento de volteo $M_{v o}$ versus la distorsión global $D_{r}$. Los valores de $M_{v o}$ se presentan sin dimensiones dividiéndolos entre su valor máximo, $M_{v o \text { max }}$. Estos resultados permiten valuar de manera aproximada la ductilidad global de desplazamientos de la estructura, $\mu$, la que resulta igual a 2.6. Este parámetro da una idea del comportamiento inelástico global de la estructura.

El valor calculado para la distorsión global $D_{r}$ con el programa ETABS y las NTCS fue $0.86 \%$. La Fig. 8 muestra valores para $D_{r}$ obtenidos con el programa de análisis dinámico no lineal, el valor máximo para este parámetro fue $1.44 \%$, es decir 1.7 veces el calculado con las NTCS. La distorsión de entrepiso máxima calculada con las NTCS fue $1.2 \%$, el valor máximo calculado con el análisis dinámico no lineal fue $2.54 \%$, correspondiente al cuarto nivel, como muestra el valor para $d_{r \text { Max }}$ en la Tabla 3, es decir 2.1 veces el calculado con las NTCS. Estos resultados confirman de manera numérica lo ilustrado en la Fig. 2, y lo anteriormente comentado de que el empleo de los espectros de diseño del cuerpo principal de las NTCS lleva a subestimar los valores de desplazamientos de estructuras. 


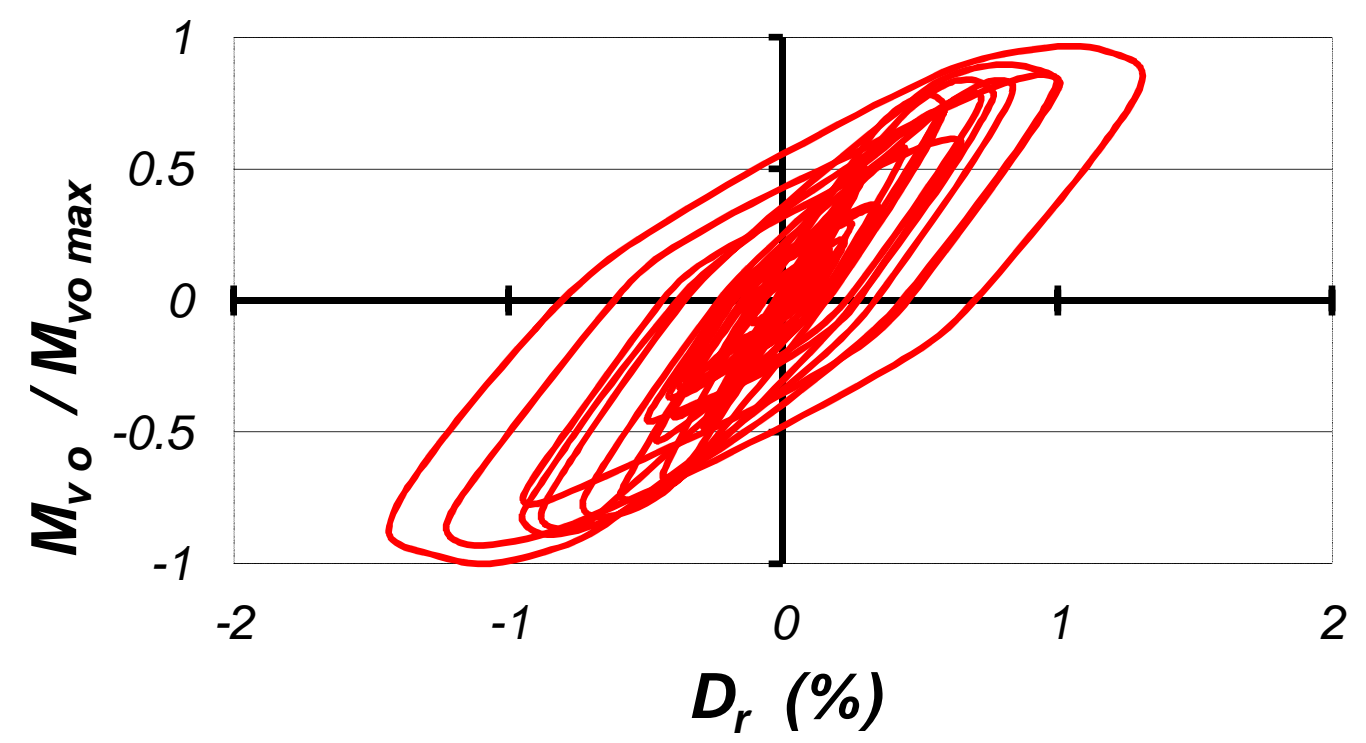

Figura 7. Momento de volteo-Distorsión global obtenido del análisis dinámico no lineal para el marco de 12 niveles.

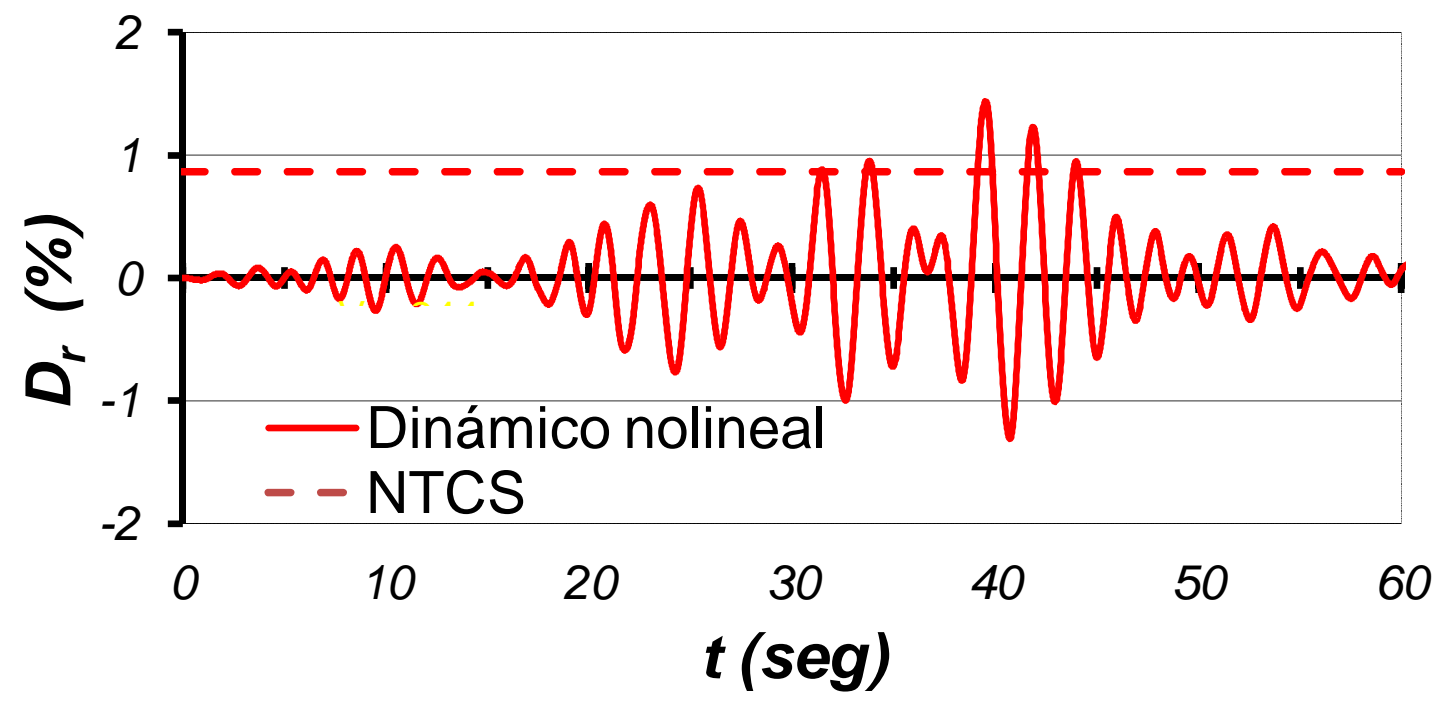

Figura 8. Distorsión global en el tiempo obtenido del análisis dinámico no lineal y con las NTCS

En la interpretación de estos resultados de evaluación de desplazamientos calculados con el procedimiento de las NTCS y los de un análisis dinámico no lineal se debe considerar que estos últimos desplazamientos se valuaron empleando para $\xi$ un valor igual a 5\%, y en cualquier caso de suelo, estos desplazamientos serían menores que los calculados con un análisis dinámico no lineal empleando para $\xi$ valores iguales o menores que 2\%, como lo sugieren algunos autores (Panagiotou, 2008; Martinelli y Filippou, 2009). Por ejemplo para el caso del edificio en estudio, cuando en el programa de análisis dinámico no lineal se consideró el caso de $\xi=2 \%$ en todos los modos, la distorsión global máxima en este caso fue igual a $1.72 \%$, es decir 2.0 veces el calculado con las NTCS, y la distorsión máxima de entrepiso calculada ocurrió en el cuarto nivel y fue igual a $2.95 \%$, es decir 2.5 veces el calculado con las NTCS. 
Con base en la evaluación anterior, se propone estipular espectros para diseño sísmico en los que el ingeniero considere de manera explícita el factor de sobrerresistencia, como por ejemplo se hace en el Apéndice de las NTCS y en el Manual de Diseño de Obras Civiles de la CFE (CFE, 2008). En lo que sigue, se presenta una propuesta de modelo para estos espectros de diseño. En el procedimiento propuesto las distorsiones de entrepiso permisibles deberán ser bastante mayores que las actualmente estipuladas en el cuerpo principal de las NTCS.

\section{Propuesta de modelo de espectros de diseño}

En esta parte del trabajo se proponen modelos de espectros de diseño de resistencia y de desplazamientos considerando el comportamiento inelástico de las estructuras, con la característica de que en su cálculo no se emplean espectros elásticos de respuesta. Este criterio es particularmente útil para estructuras en suelos blandos, donde las reducciones de ordenadas espectrales elásticas no siguen las conocidas reglas de reducción típicas en suelo firme (Meli y Ávila, 1989).

Para valuar la respuesta espectral inelástica, se empleó el modelo de Takeda modificado (Carr, 2002), con el fin de considerar el comportamiento típico de estructuras de concreto reforzado, en lugar de considerar el modelo elastoplástico, comúnmente empleado para obtener espectros de respuesta. El parámetro de rigidez de descarga, $\alpha$, Ec (10), se consideró igual a 0.5. El parámetro de rigidez de carga, $\beta$ (Carr, 2002), se consideró igual a 0.5.

La Fig. 9 muestra espectros de resistencia que se proponen para su empleo en suelos blandos del DF, y corresponden al modelo de Takeda, valores de $\mu$ iguales a 1,2 y 4 , y considerando para $\xi$ el valor $2 \%$, este último valor se empleó tomando en cuenta las recomendaciones de otros autores anteriormente mencionadas. Se observa que en la zona de periodos menores que alrededor de $1.3 \mathrm{seg}$, las ordenadas espectrales inelásticas son bastante parecidas, y en algunos casos incluso son cercanas a las ordenadas elásticas. La Fig. 10 muestra valores de desplazamientos espectrales, $S_{d}$, considerando el modelo de Takeda, $\xi$ igual a $2 \%$, y valores de $\mu$ iguales a 1,2 y 4 . Se aprecia que en la zona de periodos menores que $1.6 \mathrm{seg}$, a mayor ductilidad mayores demandas de desplazamientos, por lo contrario, para periodos mayores que $1.6 \mathrm{seg}$, a mayor ductilidad menores demandas de desplazamientos.

$$
\text { Takeda, } \mu=1,2,4, \xi=2 \%
$$

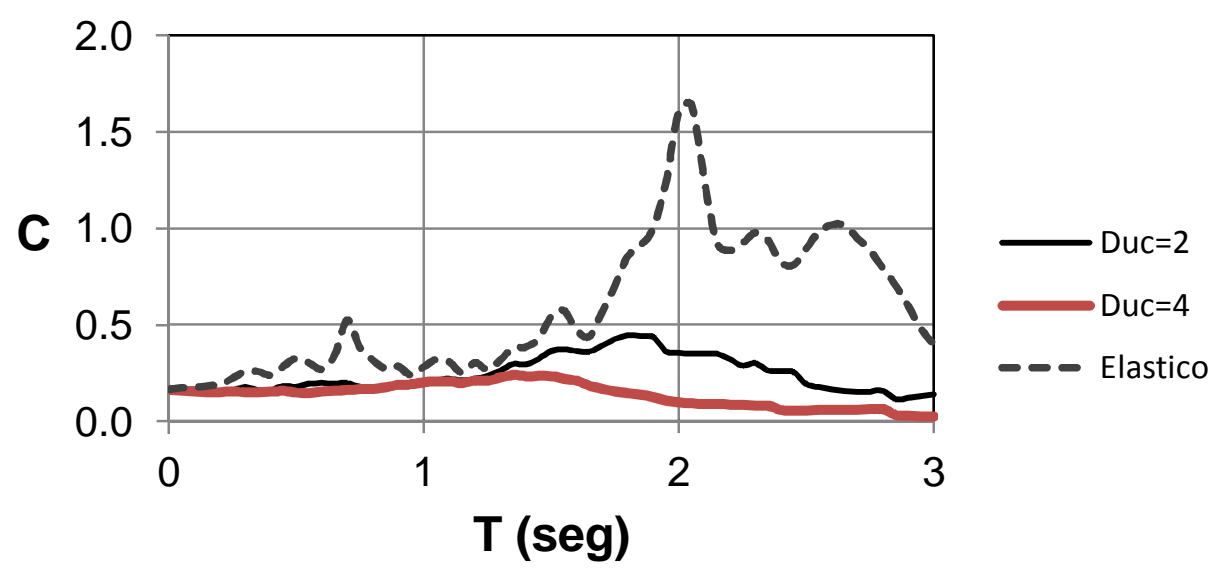

Figura 9. Espectros de aceleraciones para el registro SCT, modelo de Takeda, $\xi=2 \%, \mu=1,2,4$. 


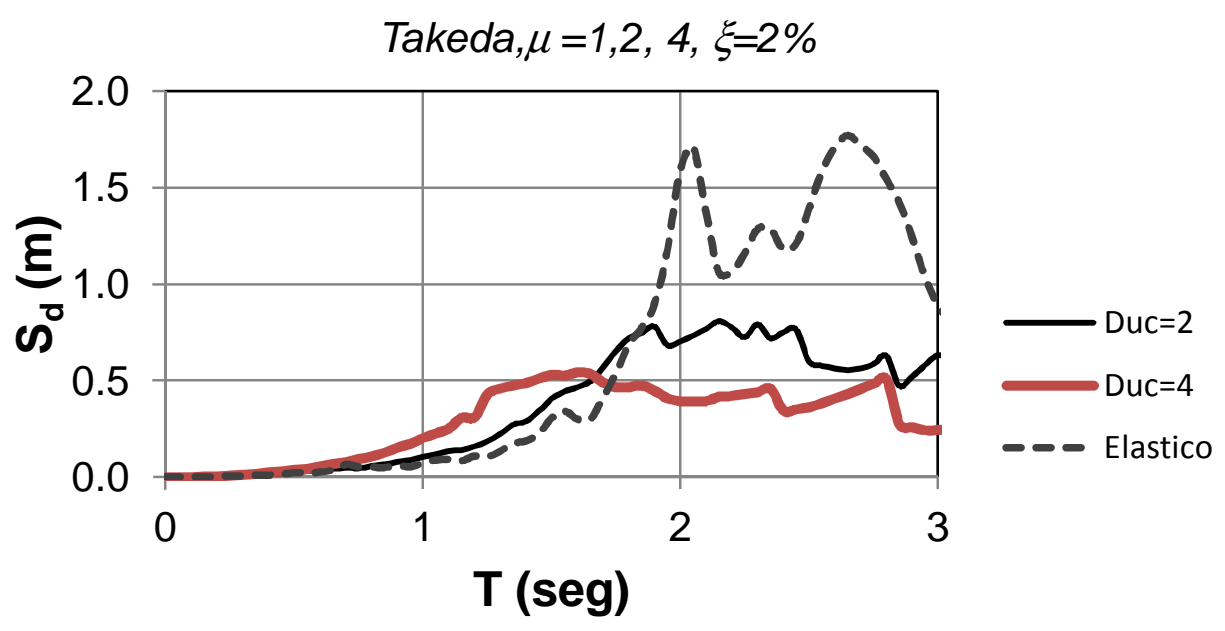

Figura 10. Espectros de desplazamientos para el registro SCT, modelo de Takeda, $\xi=2 \%, \mu=1,2$ y 4 .

Los resultados anteriores indican que las reducciones de ordenadas espectrales elásticas debido a comportamiento inelástico es función no solo de los periodos de la estructura, sino también del periodo dominante del terreno, $T_{s}$, características que no son tomadas en cuenta en los valores del factor de reducción $Q$ ' especificados por el cuerpo principal de las NTCS. Estas características sí son tomadas en cuenta en los valores del factor de ductilidad $Q$ ' especificados por el Apéndice A de las NTCS; sin embargo, en algunos casos estos valores no son congruentes con los que se muestran en este trabajo. Para ilustrar estas diferencias, se comenta en lo que sigue un ejemplo de cálculo del factor $Q$ ' de acuerdo con el Apéndice A, en el cual se consideran estructuras ubicadas en el tipo de suelo donde se obtuvo el registro SCT mencionado, y para el cual se supone un valor de $T_{s}$ igual a $2.0 \mathrm{seg}$. Los periodos característicos $T_{a} \mathrm{y}$ $T_{b}$ especificados por el Apéndice A para este valor de $T_{s}$ son iguales a $1.2 \mathrm{seg}$ y $2.4 \mathrm{seg}$, respectivamente. Para obtener las ordenadas espectrales elásticas especificadas por el Apéndice A intervienen los parámetros $k$ y $a_{o}$, los que de acuerdo con este Apéndice para el caso $T_{s}=2$ seg les corresponden los valores 0.35 y 0.25 , respectivamente. Con estos valores e ignorando el efecto de interacción suelo-estructura, las ordenadas elásticas del espectro de aceleraciones, $a$, para el caso $T_{s}=2$ seg se obtienen como:

$$
\begin{array}{ll}
a=0.25+0.95 \frac{T}{T_{a}} & \text { si } T<T_{a} \\
a=1.2 & \text { si } T_{a} \leq T<T_{b}
\end{array}
$$

Para sencillez del ejemplo, aquí no se comentan los resultados para la zona de periodos $T>T_{b}$. De acuerdo con el Apéndice A, para este ejemplo el factor de ductilidad, $Q$ ', en función del comportamiento sísmico $Q$ se obtiene como:

$$
\begin{aligned}
& Q^{\prime}=1+1.69(Q-1) \frac{T}{T_{a}} \quad \text { si } T \leq T_{a} \\
& Q^{\prime}=1+1.69(Q-1) \quad \text { si } T_{a}<T \leq T_{b}
\end{aligned}
$$


El cómputo de las ordenadas de aceleraciones de diseño con las Ecs (13) a la (16) para el caso del ejemplo en estudio en la zona de periodos $T<T_{a}$ y considerando los valores de $Q$ de 2 y 4 , lleva a valores de ordenadas espectrales reducidas de diseño con valores diferentes, lo que haciendo la analogía del parámetro $Q$ con $\mu$ no es congruente con los valores de las ordenadas de aceleraciones espectrales inelásticas mostradas en la Fig. 9. En consecuencia, tampoco los desplazamientos espectrales de diseño serían congruentes con las tendencias de la respuesta mostrada para $S_{d}$ en la Fig. 10 para la zona de periodos $T<T_{a}$. Estos resultados sugieren la dificultad de lograr en estructuras sobre suelo compresible, ordenadas espectrales reducidas de diseño empleando ordenadas espectrales elásticas, de manera que sean congruentes con las que se obtendrían de espectros de respuesta inelásticos.

Los resultados anteriores también indican que resultados de estudios de zonificación para fines de diseño en los que se emplee solo espectros elásticos, serían incompletos, ya que es necesario tomar en cuenta factores de reducción que dependen no sólo del periodo del terreno, sino también del periodo fundamental de la estructura. La variabilidad de esta dependencia sugiere la conveniencia de especificar directamente espectros de diseño inelásticos evitando el uso de reducciones a ordenadas espectrales elásticas.

Se debe observar que las ordenadas espectrales de la Fig. 9 corresponden a la resistencia $C_{y}$ y de acuerdo con la Ec (6), para definir la resistencia de diseño, $C_{s}$, es necesario estipular un valor para $R$, para lo cual como aproximación se podrían emplear los valores que se dan para este parámetro en el Apéndice A.

\section{Empleo de espectros de sitio}

El Apéndice A de las NTCS permite tomar en cuenta de manera explícita los efectos de sitio, con lo que es posible emplear ordenadas de espectros de diseño que pueden ser diferentes a las estipuladas en el cuerpo principal de las NTCS. Se debe mencionar que el Apéndice A especifica que estos resultados se deben emplear con los requisitos de diseño de este Apéndice. Sin embargo, la realidad es otra, como se puede apreciar en la práctica de diseño sísmico de algunos edificios importantes en el país, en la que generalmente el propietario o ingeniero estructural solicita un estudio de espectros de sitio y de acuerdo con el Apéndice A genera espectros de diseño, o simplemente solicita a algunos especialistas en el área, espectros de diseño para el sitio. En los casos que estos espectros tengan ordenadas espectrales menores que las correspondientes al cuerpo principal de las norma, entonces emplea estas ordenadas menores para el diseño sísmico del edificio o estructura, siguiendo los pasos estipulados en este cuerpo principal, es decir sin emplear el Apéndice A, lo cual no es lo estipulado en la norma.

Esta práctica de empleo de espectros de sitio debe de cambiar. Se sugiere que para el empleo de espectros de sitio se deben especificar restricciones que impidan abusar de su empleo, como por ejemplo especificar que las ordenadas espectrales que se obtengan con espectros de sitio no deban ser menores que el $85 \%$ de los valores especificados en el cuerpo principal. También sería conveniente que para la aceptación del empleo de espectros de sitio, se requiera la aprobación de un comité técnico especializado que dependa de las autoridades del Distrito Federal.

\section{EL DISEÑO SÍSMICO DE EDIFICIOS EN ZONAS DE ALTA SISMICIDAD EMPLEANDO Q= 2}

Las NTCS permiten en zonas de alta sismicidad en el DF el empleo de estructuras diseñadas con $Q$ igual a 2, donde $Q$ es el factor de comportamiento sísmico. Algunos ejemplos de zona de alta sismicidad serían la zona III en el DF, o parte de la costa del Pacífico. Es conocido que los requisitos de 
confinamiento en zonas de posible formación de articulaciones plásticas en estructuras diseñadas con $Q$ igual a 4, así como requisitos de regularidad de la estructura, son bastante más rigurosos que los requisitos respectivos para el caso de $Q$ igual a 2. Además, es conocido que la práctica de permitir estructuras no dúctiles en zonas de alta sismicidad la emplea también la versión anterior del Manual de la CFE (1993) y su última versión (CFE, 2008), así como reglamentos anteriormente mencionados para zonas sísmicas fuera del DF.

Esta práctica de emplear estructuras no dúctiles en zonas de alta sismicidad es bastante diferente a la de por ejemplo en zonas de alta sismicidad en los Estados Unidos, donde de acuerdo con el reglamento respectivo, en esas zonas no se permite el empleo de estructuras no dúctiles, es decir las que corresponderían a $Q$ igual a 2 de nuestro reglamento. Por ejemplo, en zonas de alta sismicidad de Estados Unidos, para el caso de estructuras de concreto sólo se permite el diseño de estructuras que cumplan el Capítulo 21 del ACI-318 (ACI 318-11), en el que se basa la sección 7 (Marcos Dúctiles) de las NTCC. Estas disposiciones reglamentarias de no permitir estructuras no dúctiles en zonas de alta sismicidad de Estados Unidos se basa en la incertidumbre inherente en la predicción de la demanda de acciones sísmicas. Como ejemplo de esta incertidumbre se puede mencionar el caso de las acciones sísmicas experimentadas en 1985 en el DF, las que fueron bastante mayores que las acciones sísmicas de diseño estipuladas en el reglamento vigente en el DF en esa época. El problema es aún mayor si se considera el comportamiento observado en algunas estructuras diseñadas con $Q$ igual a 2 , como se muestra a continuación.

En nuestro país, en estructuras diseñadas con $Q$ igual a 2 se permite soldar las barras de refuerzo en zonas de posible formación de articulaciones plásticas de elementos estructurales, como trabes, columnas y muros. En una gran mayoría de estructuras de concreto reforzado en México se emplea el acero de refuerzo que especifica la normativa mexicana NMX-C-407-ONNCCE-2001 (Norma Oficial Mexicana, 2001), la cual prácticamente sigue las especificaciones ASTM A615 (ASTM, 2009). Se debe mencionar que el contenido de carbono en la composición química de la barra de refuerzo es el responsable de los cambios en la microestructura en la zona afectada por el calor en el proceso de soldadura y de la dificultad para la operación de ésta. El contenido de carbono de las barras de refuerzo producidas en México es alto, lo que lleva a que las barras soldadas tengan poca capacidad de deformación. Un problema adicional que incide en la seguridad estructural de elementos de concreto reforzado en los que se emplean barras soldadas es la poca preparación de los soldadores y la deficiente supervisión del proceso, el cual se realiza generalmente en el lugar de la obra.

Investigaciones realizadas en el Instituto de Ingeniería de la UNAM (Rodríguez y Rodríguez Asabay, 2006) han indicado que el soldar varillas de refuerzo que comúnmente se emplean en México lleva a una condición frágil en las varillas, es decir sin la capacidad de deformación necesaria durante un sismo fuerte o tal vez mediano. La Fig. 11 (Rodríguez y Rodríguez Asabay, 2006) muestra curvas esfuerzo-deformación obtenidas de ensayes a tracción de barras de refuerzo sin soldar de diámetros 1", 1-1/4" y 1-1/2" (No 8, No 10 y No 12, respectivamente), producidas en el país. La Fig. 12 (Rodríguez y Rodríguez Asabay, 2006) muestra resultados de este tipo de ensayes pero para barras soldadas de diámetro No 10 soldadas con dos variantes, bisel en posición horizontal (B1), y bisel en posición vertical (B2), cumpliendo los requisitos de precalentamiento para la soldadura de elementos que especifica la ANSI/AWS D1.4-98 (AWS, 1998), y empleando electrodos de alta resistencia E90. Los resultados de la Fig. 11, correspondientes a barras sin soldar indican que éstas tienen capacidad de deformación en tracción con valores en el intervalo 0.10 a 0.12 . Los resultados de la Fig. 12 evidencian que las barras soldadas llegan a su capacidad de deformación en tracción para valores en el intervalo 0.015 y 0.03 , es decir en algunos casos las capacidades de deformación en tracción de barras sin soldar sería bastante mayores que las capacidades de las barras soldadas. 


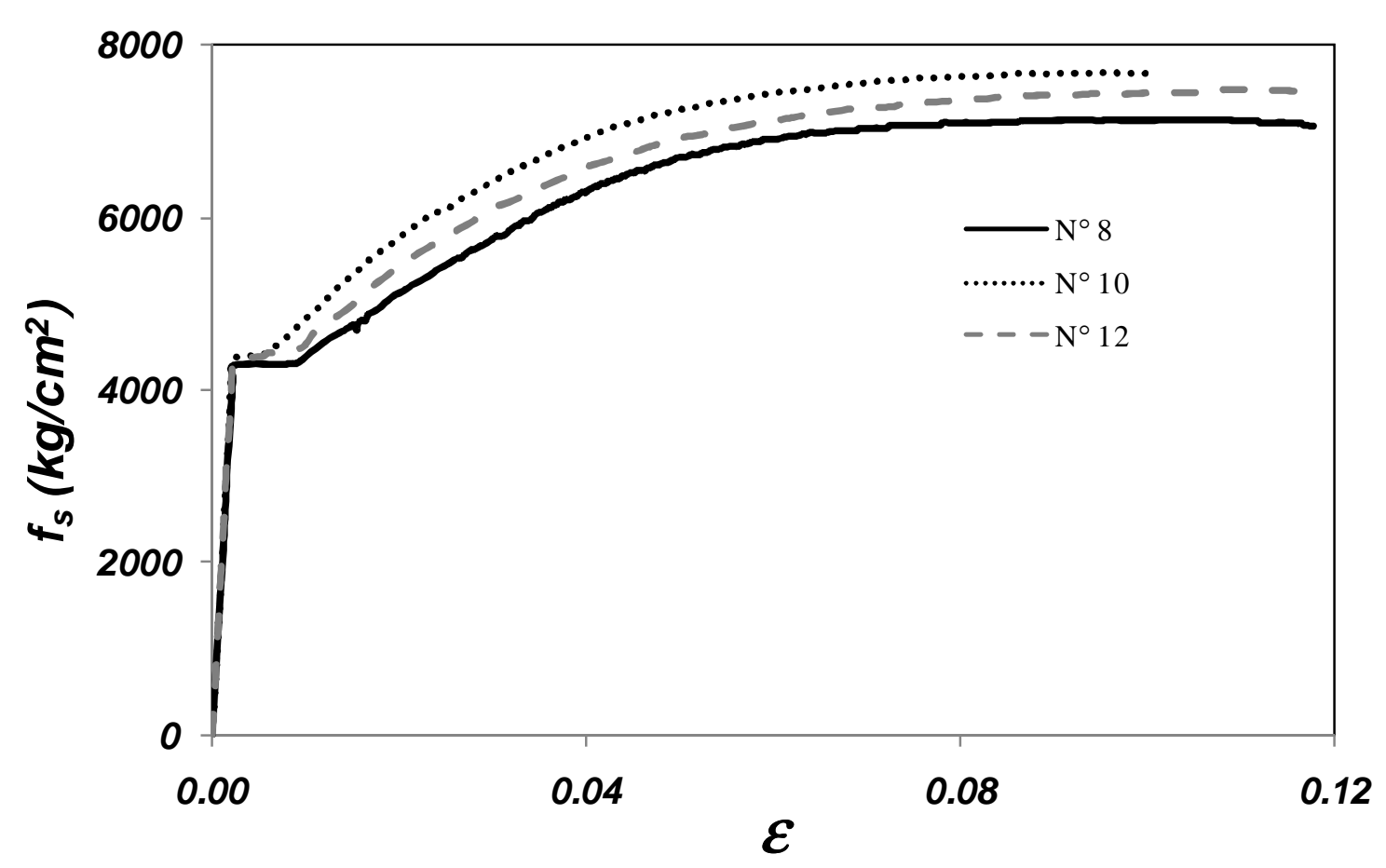

Figura 11. Curvas esfuerzo-deformación obtenidas de ensayes a tracción en barras de refuerzo sin soldadura (Rodríguez y Rodríguez Asabay, 2006)

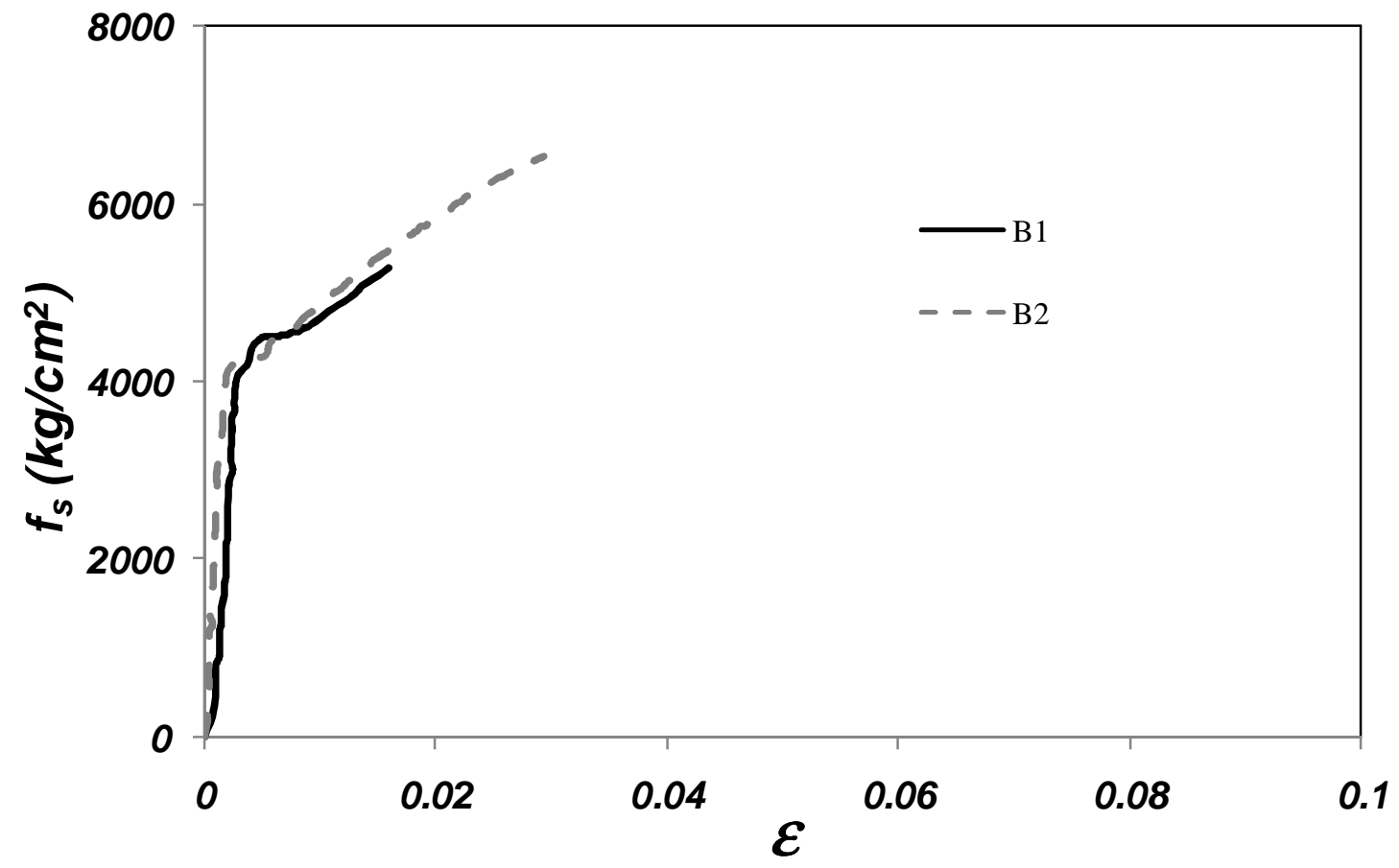

Figura 12. Curvas esfuerzo-deformación obtenidas de ensayes a tracción en barras de refuerzo soldadas No 10, soldadura con bisel tipos B1 y B2 (Rodríguez y Rodríguez Asabay, 2006) 
Zermeño et al (1990) ensayaron, hace más de dos décadas, conexiones trabe-columna ante cargas laterales cíclicas reversibles, donde la trabe era prefabricada de concreto. Sus resultados no fueron analizados en su totalidad en su momento, y han sido estudiadas recientemente (Rodríguez y Torres, 2012). La continuidad del refuerzo del lecho inferior de la trabe prefabricada se lograba soldando en sitio, con pequeños tramos de barras de refuerzo, placas de acero estructural embebidas en el extremo de la trabe y en una ménsula en la columna. El refuerzo del lecho superior se colocaba en sitio y era continuo. La Fig. 13a muestra algunos detalles de la forma de ensaye. La Fig. 13b muestra resultados de los ensayes, en este caso ciclos de carga lateral, $V$, versus el desplazamiento lateral de la trabe en su punto de aplicación de carga, $\Delta$. La trabe falló por la fractura de los tramos de barras de refuerzo soldadas en el lecho inferior (Zermeño et al, 1990), incluso sin llegar a desarrollar su capacidad de momento positivo, como se aprecia en la Fig. 13b, evidenciando un tipo de falla frágil, con poca o nula capacidad de deformación lateral. Un comportamiento similar se observó en dos ensayes del mismo tipo de otros dos especímenes semejantes (Zermeño et al, 1990). Este tipo de respuesta no es congruente con los factores de comportamiento sísmico que se emplean para este tipo de estructuras en México, lo que sugiere el abandono inmediato de esta práctica constructiva en México.

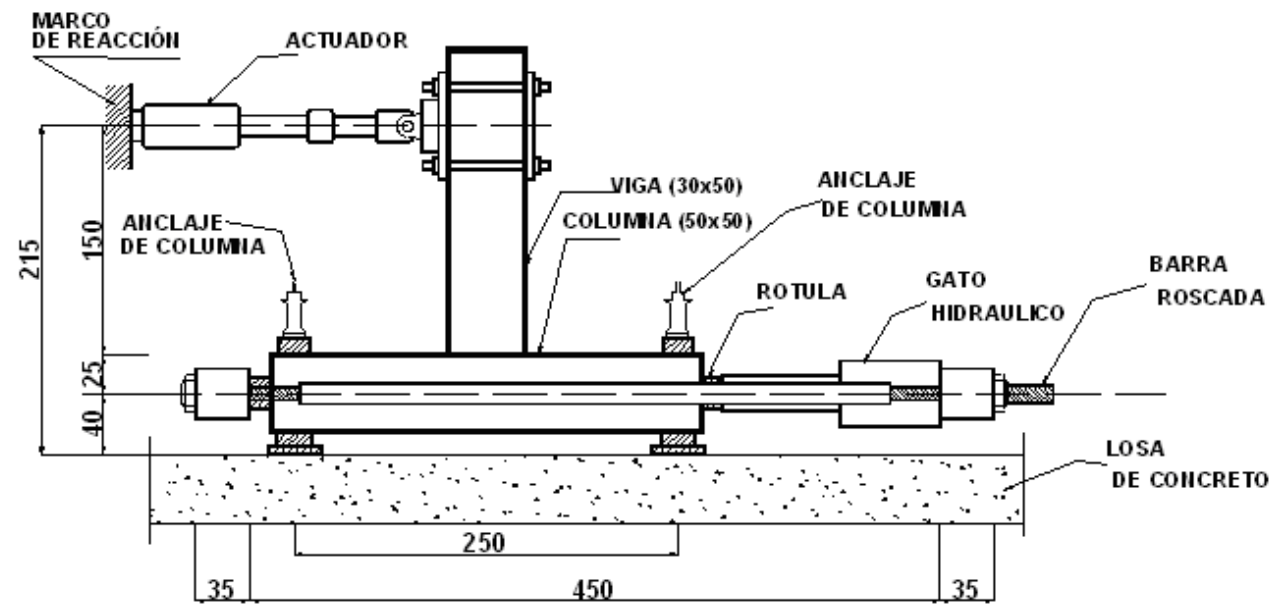

a) Marco de carga

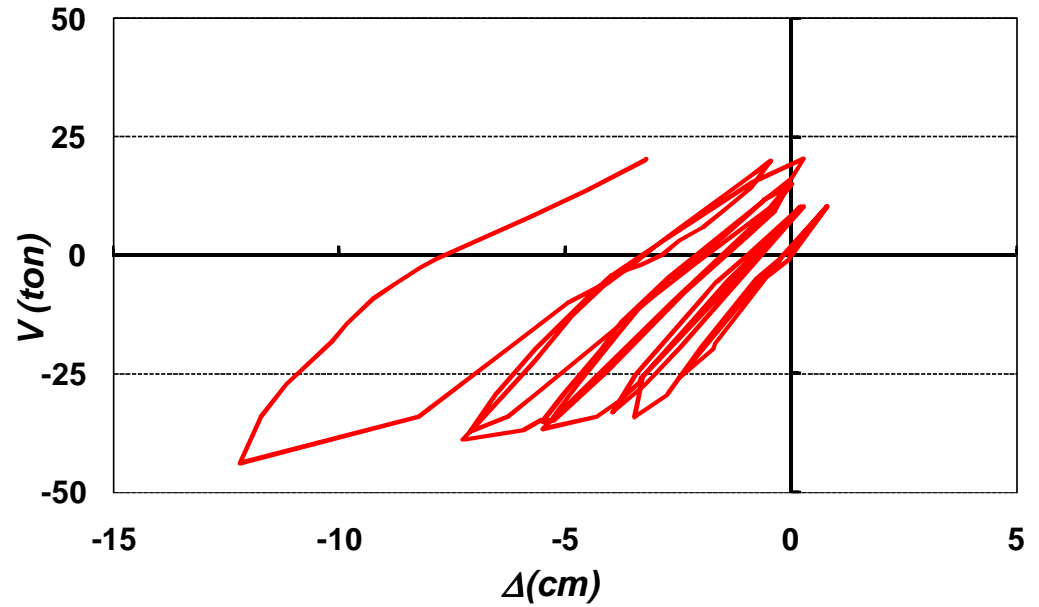

b) Ciclos carga lateral-desplazamiento medidos en ensaye.

Figura 13. Marco de carga y curvas carga lateral-desplazamiento obtenidas en ensayes ante cargas laterales de una conexión trabe-columna prefabricada (Zermeño et al., 1990) 
El problema de la fragilidad de las barras de refuerzo soldadas en zonas de alta sismicidad en México también existe en las barras de refuerzo en estructuras de concreto coladas en sitio, cuando se lleva a cabo esta práctica en zonas críticas de una estructura. Sin embargo, este problema es particularmente importante en la construcción de estructuras prefabricadas de concreto reforzado en zonas sísmicas del país. Esto se debe a que la unión de elementos prefabricados en nuestro país generalmente se lleva a cabo soldando placas y/o barras de refuerzo en las zonas de posible formación de rótulas plásticas en trabes.

Existen soluciones alternativas a esta mala práctica constructiva, como es por ejemplo el empleo de estructuras prefabricadas de concreto reforzado con base en el concepto de emulación, el cual no utiliza soldadura, y ha sido empleado en países sísmicos como Japón, Chile y Nueva Zelandia. En los terremotos recientes del 23 de febrero 2010 en Chile, y 22 de febrero 2011 en Christchurch, Nueva Zelandia, el comportamiento observado de este tipo de estructuras prefabricadas ha sido satisfactorio. En general el comportamiento de las estructuras prefabricadas en el terremoto mencionado de Christchurch, ha sido satisfactorio (Fleischman et al., 2012; Kam y Pampanin, 2011), aún cuando en algunos casos se ha observado fractura del acero de refuerzo en diafragmas para resistir las fuerzas sísmicas actuantes en el plano de éstos. El tipo de estructura prefabricada que emplea el concepto de emulación recientemente ha sido estudiado en un edificio ensayado en mesa la vibradora de la UNAM (Rodríguez et al. 2012), con resultados favorables, los que sugieren la conveniencia de su empleo inmediato en el país. Sin embargo, se debe mencionar que en este edificio se observó la fractura del acero de refuerzo en los diafragmas del edificio debido a las fuerzas sísmicas actuantes en el plano de éstos. Esto se debió a que este refuerzo, como se hace en la práctica constructiva en el país, no se diseñó para resistir fuerzas sísmicas.

Por las razones arriba descritas, se propone que en zonas de alta sismicidad en México no se permita el empleo de estructuras no dúctiles, es decir diseñadas con $Q$ igual a 2, y sólo se debiera permitir en esas zonas el empleo de estructuras diseñadas con $Q$ igual a 4 o en su caso igual a 3.

\section{EL PROBLEMA DE LA VALUACIÓN DE LA RIGIDEZ LATERAL DE ESTRUCTURAS}

Con el avance en la computación electrónica, es común encontrar diseños sísmicos de edificios importantes en los que prácticamente la memoria de cálculo consiste en innumerables hojas donde la computadora envía sus resultados. Además, muchos de quienes llevan a cabo estos ejercicios de cómputo tienen la idea equivocada de que por ser bastante elaborados son también precisos. Para agravar el problema, el proceso de diseño, es decir el detallado del refuerzo, cantidad y distribución de éste, es una etapa muchas veces efímera en el proceso total de análisis y diseño, incluso en algunos casos también es resultado de otro ejercicio de cómputo electrónico, donde el ingeniero pierde el significado físico de lo que está ejecutando. Se olvida que durante un terremoto, el comportamiento de la estructura depende de manera importante del mencionado detallado del refuerzo.

El problema es aún mayor, debido a que los valores de momentos de inercia que estipulan algunos reglamentos de construcción, incluyendo las NTCC y ACI 318-11 (ACI 318-11, 2011), son cuestionables y sobrestiman por mucho la rigidez de elementos estructurales, como se muestra a continuación, y por tanto en los análisis de estructuras se estaría sobrestimando sus rigideces laterales. Esto pone en duda la precisión en el análisis y diseño sísmico de edificios con la que muchos ingenieros están convencidos.

En este trabajo se muestran resultados del análisis de resultados de una parte de la base de datos empleada por Restrepo y Rodríguez (2012a) para la evaluación de la capacidad resistente a flexión de columnas de concreto reforzado, obtenida en ensayes ante cargas laterales cíclicas del tipo reversible 
llevados a cabo en diversos laboratorios del mundo. La base de datos analizada en este trabajo corresponde a 18 columnas de concreto reforzado de sección rectangular, y fue seleccionada de manera rigurosa, considerando sólo los casos de especímenes representativos de columnas en estructuras. Por ejemplo, de la base de datos de columnas existente, obtenida del PEER (Berry et al., 2004), no se consideraron los casos en los que por condiciones de borde de los especímenes, no se representaba de manera adecuada las condiciones de penetración de fluencia del acero de refuerzo longitudinal, como son los casos en los que las columnas se ensayan en doble voladizo (Berry et al., 2004).

Resultados de la evaluación de la base de datos seleccionada se muestran en la Fig. 14 (Restrepo y Rodríguez, 2012b). Estos resultados corresponden a valores del cociente entre la rigidez efectiva, $E I_{e}$, y la rigidez bruta, $E I_{g}$. El parámetro $E$ es el módulo de elasticidad del concreto y los parámetros $I_{e}$ y $I_{g}$ son el momento de inercia efectivo obtenido de resultados de ensayes ante cargas laterales de columnas en laboratorio y el momento de inercia de la sección bruta, respectivamente. La rigidez efectiva, $E I_{e}$, se valuó con las curvas experimentales momento-desplazamiento lateral empleando la rigidez secante a 0.75 $M_{M A X}$, donde este último parámetro es el momento máximo medido. Los resultados en la Fig. 14 para los valores del cociente $E I_{e} / E I_{g}$ se muestran en función de la relación de carga axial $P /\left(A_{g} f_{c}^{\prime}\right)$, donde $P$ es la carga axial actuante, $A_{g}$ es el área transversal de la columna y $f_{c}^{\prime}$ es la resistencia a compresión del concreto medida en los cilindros de prueba correspondientes a los especímenes. Se aprecia en estos resultados que hay una fuerte correlación entre la rigidez efectiva y la relación de carga axial. Además, para valores de esta relación menores que 0.3 , valores que se pueden considerar típicos en columnas de edificaciones de concreto, los valores de la rigidez efectiva son menores que $0.4 I_{g}$. Elwood y Eberhard (2009) han analizado una base de datos de ensayes ante cargas laterales de columnas de concreto reforzado, y han encontrado resultados comparables con los que aquí se comentan. Se debe mencionar que los autores emplearon una definición de rigidez efectiva diferente a la empleada por Elwood y Eberhard (2009) ya que estos autores emplearon el concepto de rigidez secante a la primera fluencia, definida como el punto en el cual fluye el refuerzo por primera vez o el concreto alcanza la deformación máxima de 0.002 .

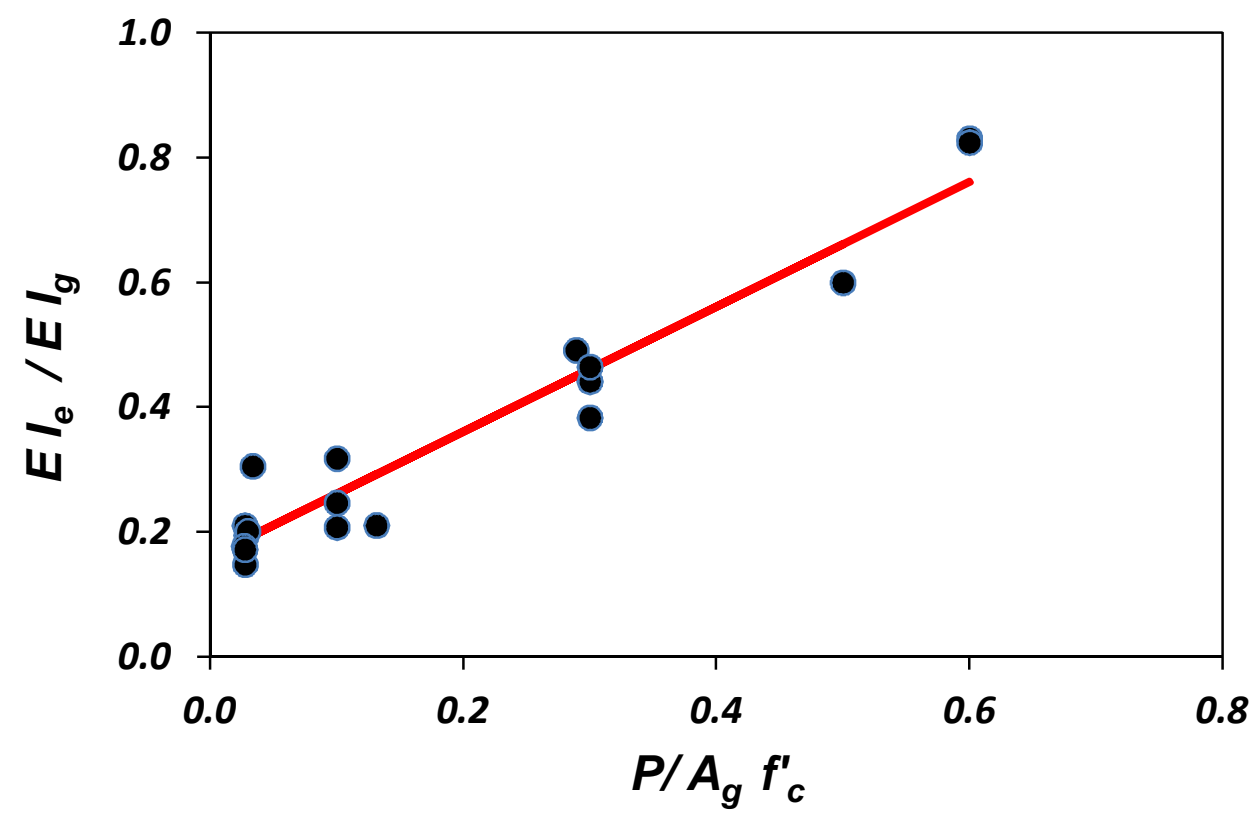

Figura 14. Rigidez efectiva en columnas rectangulares de concreto reforzado en función de la relación de carga axial, resultados experimentales y calculados (Restrepo y Rodríguez, 2012b) 
Con base en los resultados mostrados en la Fig. 14, se propone la siguiente expresión para valuar el momento de inercia efectivo, $I_{e}$, de columnas rectangulares, de concreto reforzado:

$$
I_{e}=\left(0.16+\frac{P}{A_{g} f_{c}^{,}}\right) I_{b}
$$

Los resultados de aplicar la Ec (17) para la base de datos seleccionada se muestra en la Fig. 14. El promedio del cociente entre el valor experimental de $I_{e}$ y el calculado con la Ec (17) es igual a 1.01, y el coeficiente de variación, CV, es 0.19 . Este valor de CV se debe principalmente a que la Ec (17) no toma en cuenta otros factores que intervienen en la rigidez efectiva de columnas como son la cuantía de refuerzo y la relación de esbeltez de columnas (Restrepo y Rodríguez, 2012b).

Estos resultados indican que las NTCC, y también el ACI 318-11, en estos casos estarían sobrestimando la rigidez de estas columnas en más del $100 \%$, ya que especifican el valor de $I_{g}$ como valor a emplear en el análisis. Es de interés ver en los resultados experimentales de la Fig. 14 que la rigidez efectiva en columnas con baja relación de carga axial puede alcanzar valores muy bajos, del orden del $20 \%$ del valor de la rigidez correspondiente a la sección bruta, y que sólo para valores de la relación de carga axial mayores que 0.5 se tendría rigideces efectivas mayores que alrededor del $50 \%$ de la rigidez correspondiente a la sección bruta. Estos resultados difieren por mucho de los valores de momentos de inercia para columnas que especifican las NTCC y ACI 318-11, y ponen en evidencia la falta de precisión que se tendría en el análisis y diseño sísmico de edificios empleando estas normativas, aún con programas de cómputo elaborados. Se sugiere revisar los valores de momentos de inercia que se estipulan en las normativas actuales en México. Para el caso de columnas rectangulares de concreto reforzado se recomienda emplear la Ec (17) para valuar la rigidez efectiva.

\section{EL PROBLEMA DEL DISEÑO SÍSMICO DE SISTEMAS DE PISO DE EDIFICIOS PARA FUERZAS SÍSMICAS HORIZONTALES Y EL DISEÑO SÍSMICO DE APÉNDICES}

La intención de la sección 8.4, “Apéndices", de las NTCS es la de valuar las fuerzas sísmicas que actúan en elementos tales como tanques, apéndices o losas en edificios, para lo cual especifica valores de aceleraciones absolutas horizontales que actúan en estos elementos estructurales. La aceleración absoluta horizontal en el nivel $i$ del edificio multiplicada por la masa del nivel $i$ o por la masa del apéndice rígido define la fuerza horizontal actuante en la losa del nivel $i$ o en el apéndice, respectivamente.

Llama la atención que las NTCS trate de manera oscura el diseño sísmico de losas para fuerzas inerciales horizontales, ya que al final de la sección 8.4, y con una redacción deficiente, esta sección estipula "se incluyen, asimismo..." a "las losas que transmiten fuerzas de inercia de las masas que la soportan". Esto ha llevado a que ingenieros estructurales, incluso en proyectos relevantes, interpreten que esta sección de las NTCS no es aplicable al caso del diseño sísmico de diafragmas para fuerzas sísmicas horizontales. En particular, son preocupantes los casos de diafragmas de edificios construidos con "losacero", firmes colados en sitio en estructuras prefabricadas o sistemas de piso novedosos, en los cuales es común que no se haga la revisión por sismo de diafragmas para fuerzas sísmicas horizontales. Pareciera que por definición los ingenieros estructurales en México aceptan que los diafragmas en edificios no sólo son infinitamente rígidos (lo cual es un tema que aquí los autores no tocan), sino también infinitamente resistentes, y por lo tanto, ¿para qué molestarse en revisar su capacidad sísmica resistente para fuerzas sísmicas en su plano?. 
Además de la necesidad de revisar la redacción de la sección 8.4, "Apéndices", de las NTCS relacionada con la observación anterior, con base en los argumentos que a continuación se exponen, se sugiere revisar el procedimiento de cálculo de las referidas fuerzas sísmicas horizontales estipulado por las NTCS.

La sección 8.4 de las NTCS especifica que las fuerzas sísmicas horizontales de diseño se deben calcular suponiendo que sobre el elemento estructural "actúa la distribución de aceleraciones que le correspondería si se apoyara directamente sobre el terreno multiplicada por"

$$
1+\frac{c^{\prime}}{a_{o}}
$$

"donde $c$ ' es el factor por el que se multiplican los pesos a la altura de desplante del elemento cuando se valúan las fuerzas laterales sobre la construcción".

La Ec (18) tiene limitaciones, las que como se muestra en este trabajo, no permiten valuar de manera satisfactoria los valores de las aceleraciones absolutas horizontales actuantes en los niveles de un edificio. Una limitación es que, como se ha comentado en este trabajo, el factor c' está asociado a la resistencia $C_{s}$, la cual es diferente a la resistencia $C_{y}$, necesaria para la evaluación de las aceleraciones de piso. Además, de acuerdo con principios básicos de la dinámica estructural, la fuerza sísmica horizontal que debe resistir el diafragma en el nivel $i$ de un edificio, es el producto de la masa en este nivel por la aceleración horizontal absoluta del nivel. Esta aceleración no está relacionada con la Ec (18), como se puede deducir de la conocida ecuación de equilibrio dinámico de una estructura.

Lo anterior sugiere que el procedimiento de cálculo de aceleraciones horizontales máximas en estructuras que estipula la NTCS se debe corregir, para poder llevar a cabo un diseño sísmico racional de sistemas de piso para fuerzas sísmicas horizontales, o para el diseño sísmico de apéndices. Para este fin, se propone un procedimiento de diseño sísmico, el cual se muestra a continuación.

\section{Propuesta para valuar fuerzas sísmicas máximas en diafragmas y apéndices rígidos de edificios}

En la literatura (Rodríguez et al., 2002; Rodríguez et al., 2007) se menciona que para el cálculo de las fuerzas sísmicas horizontales se debe considerar el efecto de los modos superiores de manera diferente a como lo hace las NTCS, ya que como ha sido mostrado por estos autores, para el cálculo de estas fuerzas la reducción por ductilidad y sobrerresistencia se debe llevar a cabo sólo para el primer modo, lo que indica que los procedimientos actuales de diseño sísmico en el que se reducen todos los modos por el factor de reducción podrían estar del lado de la inseguridad. Estos autores han propuesto un procedimiento sencillo para valuar las aceleraciones absolutas horizontales en los niveles de un edificio, que toma en cuenta el referido efecto y que podría ser empleado en un posible cambio de la normativa que aquí se comenta.

Con base en estudios analíticos, Rodríguez et al. (2002), validados con estudios experimentales, Rodriguez et al. (2007), han propuesto, un procedimiento para valuar las fuerzas sísmicas horizontales máximas en diafragmas y apéndices rígidos de edificios. En estas referencias se muestra que el cálculo de estas fuerzas con métodos modales sólo es correcto para la respuesta elástica, ya que el comportamiento inelástico no tiene mucha influencia en las aceleraciones causadas por los modos superiores, El procedimiento que se propone en este trabajo se basa en estas referencias, la respuesta total modal se valúa con los requisitos de las NTCS, empleando la raíz cuadrada de la suma de los cuadrados de la respuesta de 
los modos, pero con la variante de que la contribución de los modos superiores se considera elástica, y la reducción por comportamiento inelástico se hace sólo para el primer modo.

El procedimiento se basa en valuar la fuerza de piso máxima en el último nivel del edificio, $F_{n}$, la cual se valúa como:

$$
\frac{F_{n}}{m_{n} g}=\sqrt{\left[\frac{\eta_{1} S_{a}\left(T_{1}, \xi\right)}{R_{M} g}\right]^{2}+\eta_{2} \ln (n) a_{o}^{2}}
$$

donde $S_{a}\left(T_{1}, \xi\right)$ es la aceleración espectral para la respuesta elástica, $T_{1}$ es el periodo fundamental de vibración del edificio, y el parámetro $m_{n}$ representa la masa del piso del nivel $n$. El factor $R_{M}$ se definió en la Ec (12), por lo que se infiere que la relación $\frac{S_{a}}{R_{M}}$ en la Ec (19) está asociada a la resistencia $C_{y}$. Cuando se emplee el Apéndice de las NTCS, $R_{M}$ se puede considerar igual al factor de comportamiento sísmico $Q^{\prime}$. Para el caso del empleo del cuerpo principal de las NTCS, de acuerdo con la Ec (6), para valuar $C_{y}$ es necesario conocer el valor de R. El parámetro $\eta_{l}$ toma en cuenta la contribución del primer modo y $\eta_{2}$ toma en cuenta la contribución de los modos superiores. En la Ec (19) se logran resultados para el límite superior con los parámetros $\eta_{1}=8 / 5$ y $\eta_{2}=1.75$. Resultados para el límite inferior en la Ec (19) se $\operatorname{logran}$ con $\operatorname{los}$ parámetros $\eta_{1}$ y $\eta_{2}$ igual a $6 / 5$ y $5 / 8$, respectivamente. Estos valores del límite superior e inferior se dedujeron a partir del empleo de modelos de vigas de flexión y cortante, respectivamente (Rodriguez et al., 2007).

Schoettler y Restrepo (2010) han mostrado que el término $\eta_{2} \ln (n)$ en la Ec (19) para el caso del límite superior se puede reemplazar por el parámetro $\eta_{3}$, definido como:

$$
\eta_{3}=1.4 \sqrt{n-1} \leq 5
$$

Con base en las Ecs (19) y (20), la expresión para $F_{n}$ que se propone a emplear con el procedimiento del Apéndice A es:

$$
\frac{F_{n}}{W_{n}}=\sqrt{\left[\frac{\eta_{1} a}{Q}\right]^{2}+\eta_{3} a_{o}^{2}}
$$

donde $W_{n}$ es el peso del último nivel del edificio, $a$ es la ordenada espectral elástica de aceleraciones estipulada en el Apéndice A. Se sugiere emplear para $\eta_{1}$ y $\eta_{3}$ los valores $8 / 5$ y el dado por la Ec (20), respectivamente, correspondientes al límite superior.

Si se emplea el cuerpo principal de las NTCS se sugiere emplear la siguiente expresión para $F_{n}$ : 


$$
\frac{F_{n}}{W_{n}}=R \sqrt{\left[\frac{\eta_{1} a}{Q^{\prime}}\right]^{2}+\eta_{3} a_{o}^{2}}
$$

donde para el factor de sobrerresistencia $R$ se sugiere el valor 2.5.

Se define $\Omega_{i}$ al factor de amplificación de aceleraciones en el nivel $i$ de un edificio, con masa $m_{i}$ en este nivel, como:

$$
\Omega_{i}=\frac{F_{i}}{m_{i} g a_{o}}
$$

Para el nivel $n$, este parámetro se valúa como:

$$
\Omega_{n}=\frac{F_{n}}{W_{n} a_{o}}
$$

Para una evaluación aproximada de $\Omega_{i}$, en este trabajo se propone para este parámetro una variación lineal, lo que lleva a la siguiente expresión

$\Omega_{i}=1+\frac{h_{i}}{H}\left(\Omega_{n}-1\right)$

Para ilustrar la aplicación de este procedimiento propuesto y su comparación con los resultados de aplicar la normativa actual, en lo que sigue se desarrolla un ejemplo para un edificio que muestra los resultados de ambos procedimientos.

\section{Ejemplo de valuación de fuerzas sísmicas horizontales en un edificio de acuerdo con la sección 8.4, “Apéndices”, de las NTCS, y con el procedimiento propuesto}

En esta parte del trabajo se muestran resultados de valuar las aceleraciones absolutas horizontales de piso, o en adelante denominadas solo aceleraciones horizontales, en el edificio de 12 niveles que se ha descrito en la primera parte de este trabajo.

La Fig. 15 muestra resultados para el marco en estudio para el factor de amplificación dinámica de aceleraciones horizontales, $\Omega_{i}$, obtenidos empleando la Ec (23) y resultados del análisis dinámico no lineal con el programa Ruaumoko, con el procedimiento de análisis y el registro de aceleraciones SCT anteriormente descritos, y considerando para el valor de la fracción de amortiguamiento crítico, $\xi$, un valor constante para todos los modos, igual a $2 \%$. En estos resultados se consideró $a_{o}=0.17$. Los valores calculados para el factor $\Omega_{i}$ se muestran para cada nivel del marco, al cual le corresponde la altura relativa $h_{i} / H$. Estas aceleraciones corresponden a los valores calculados máximos para cada nivel, y no necesariamente ocurren en el mismo instante. La Fig. 15 también muestra los valores para $\Omega_{i}$ que se obtienen aplicando los requisitos de la sección 8.4 de las NTCS, es decir empleando la Ec (18), resultados de un análisis estático, $V_{b}=0.1 \mathrm{~W}$, y $a_{o}=0.1$. Respecto a este valor para $a_{o}$ se debe mencionar que considerando el conocido registro de la SCT de 1985, el valor de la aceleración horizontal máxima del terreno para esta zona en realidad fue al menos igual a $0.17 \mathrm{~g}$, es decir mayor en un $70 \%$ que el valor considerado en las NTCS. 
También la Fig. 15 muestra resultados de aplicar para el marco en estudio el procedimiento propuesto para valuar $\Omega_{n}$, empleando las Ecs (21) y (24), con los valores para $\eta_{1}$ y $\eta_{3}$ correspondientes a los del límite superior, es decir 8/5 y el dado por la Ec (20), respectivamente. La Fig. 15 también muestra la distribución de valores de $\Omega_{i}$ en la altura del edificio empleando la Ec (25). En el empleo de la Ec (21), $a$ se valuó del espectro de aceleraciones elástico para el registro SCT, y fue igual a 0.576, y corresponde a $T_{l}=1.73 \mathrm{seg}$ y $\xi=0.05$. El valor de $Q^{\prime}$ se tomó igual al del factor de reducción $R_{M}$, el cual fue 2.19 , ver Tabla 3, y para $a_{o}$ se empleó el valor 0.17 .

Los resultados mostrados en la Fig. 15 indican que la aplicación de las NTCS para valuar las aceleraciones horizontales está del lado de la inseguridad. Los valores calculados con el análisis dinámico no lineal en algunos casos superan en más del $75 \%$ a los valores calculados con las NTCS. Por el contrario, la aplicación del procedimiento propuesto para valuar las aceleraciones horizontales llevan a una envolvente razonablemente conservadora para valuar estas aceleraciones. Se debe observar que en este ejemplo en el procedimiento propuesto se emplearon las ordenadas del espectro elástico de aceleraciones considerando para $\xi$ el valor $5 \%$, lo que indica que si en este procedimiento se emplea para $\xi$ el valor $2 \%$, llevaría a una predicción de aceleraciones con valores mayores que los mostrados en la Fig. 15.

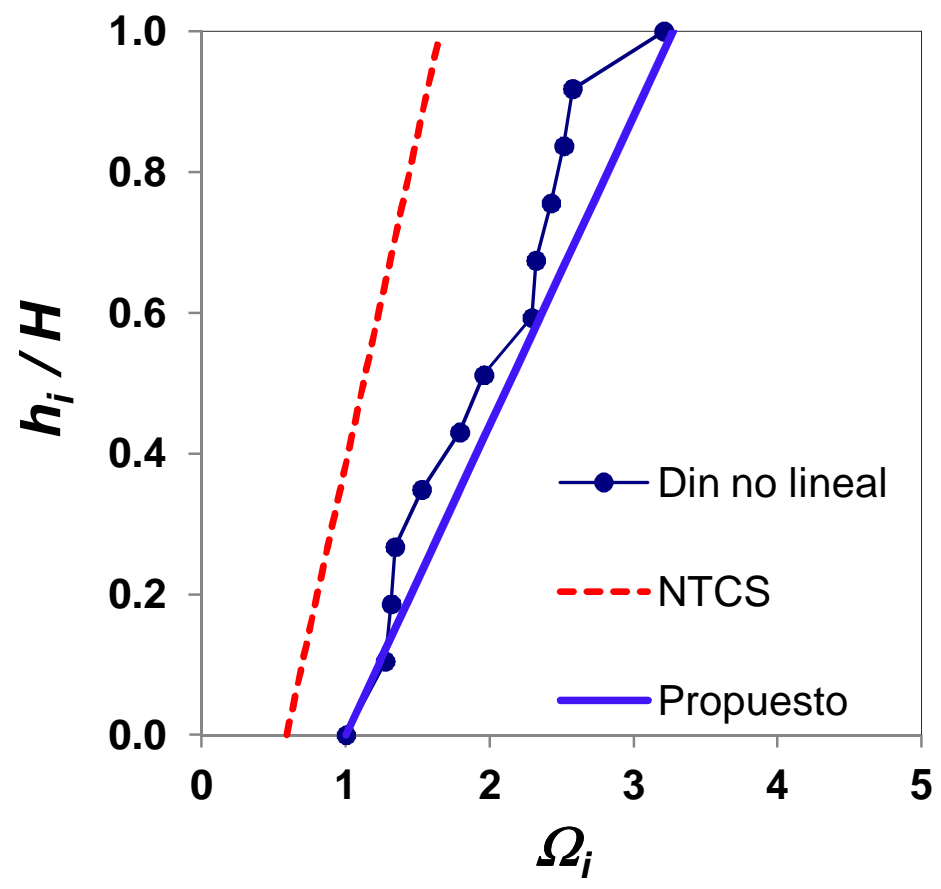

Figura 15. Factores de amplificación, $\Omega_{i}$, obtenidos del análisis dinámico no lineal, NTCS, y procedimiento propuesto.

Con el fin de mejorar la normativa actual para el DF, en lo que se refiere la sección 8.4 de las NTCS, se propone que la redacción de esta sección debe mejorar, de manera que estipule de manera clara que no sólo los apéndices y otros elementos se deben diseñar para fuerzas de inercia horizontales, sino también los diafragmas de edificios. Además, el procedimiento de diseño que se proponga para el cálculo de estas fuerzas debe emplear valores realistas de las posibles aceleraciones máximas del terreno, así como tomar en cuenta de manera racional el efecto de los modos superiores en estas fuerzas. Para tomar 
en cuenta este efecto se sugiere emplear el procedimiento de diseño propuesto por Rodríguez, et al. (2002), el cual fue adaptado en este trabajo para ser empleado con los procedimientos de diseño por sismo que estipula las NTCS del DF.

\section{EL APÉNDICE A DE LAS NTCS}

El Apéndice A de las NTCS estipula espectros elásticos de diseño que corresponden a la resistencia $C_{e}$ anteriormente definida. La resistencia de diseño $C_{s}$ se obtiene dividiendo $C_{e}$ entre el producto $Q^{\prime} R$. Aun cuando este procedimiento de diseño corrige las deficiencias observadas en este trabajo para el procedimiento de diseño sísmico especificado por el cuerpo principal de las NTCS, se considera que el procedimiento del Apéndice A se debe de revisar.

Tanto las ordenadas del espectro de aceleraciones para diseño sísmico, $a$, como el factor $Q$, especificados por el Apéndice A, dependen de, entre otros factores, del parámetro $\beta$, denominado factor de reducción por amortiguamiento suplementario para tomar en cuenta el efecto de interacción sueloestructura, el cual se especifica igual a uno cuando se ignora este efecto. De acuerdo con el Apéndice A, el parámetro $\beta$ depende de $\tilde{T}_{e}$, periodo efectivo del sistema suelo-estructura, y de la relación $\zeta_{e} / \tilde{\zeta}_{e}$, donde $\zeta_{e}$ es la fracción de amortiguamiento crítico de la estructura supuesta en base indeformable, y se especifica para este parámetro el valor 0.05. El parámetro $\tilde{\zeta}_{e}$ está relacionado con el amortiguamiento efectivo del sistema suelo-estructura, y se especifica que su valor no debe ser menor que 0.05. Además, se especifica que $\tilde{\zeta}_{e}$ depende de, entre otros parámetros, del coeficiente de amortiguamiento del suelo, que a su vez depende de "amortiguamientos viscosos" definidos como "la fuerza y el momento requeridos para producir una velocidad unitaria del cimiento en traslación horizontal y rotación, respectivamente", y cuyos valores se proporcionan en una tabla. Se observa la incongruencia entre el grado de refinamiento que se intenta emplear para definir $\tilde{\zeta}_{e}$ y la aproximación empleada para valuar $\zeta_{e}$. Se sugiere valuar los parámetros $\zeta_{e}$ y $\tilde{\zeta}_{e}$ con procedimientos con aproximaciones comparables, de preferencia simples y racionales. Se debe mencionar que los autores no afirman que no se deben emplear métodos elaborados para valuar estos parámetros, lo que sugieren es que estos métodos no sean incongruentes en lo referente a las aproximaciones empleadas en la valuación de estos parámetros.

Este intento de refinamiento para definir $a$ y $Q$ ' también contrasta con otra aproximación que se emplea en el Apéndice A. Como se ha mencionado, para definir las fuerzas sísmicas de diseño se emplea el factor $Q^{\prime} R$. Sin embargo, el Apéndice A estipula valores empíricos para el factor de sobrerresistencia $R$ (Ordaz et al., 2000), factor que no toma en cuenta características de la estructura, como grado de hiperestaticidad, número de niveles, tipo de sistema estructural, materiales, etc.

Por lo anterior, se sugiere la revisión del Apéndice A para evitar incongruencias en el grado de refinamiento que se pretende lograr para valuar diversos parámetros que intervienen en el diseño sísmico de estructuras. 


\section{CONCLUSIONES}

De los resultados mostrados en este trabajo se obtienen las siguientes conclusiones:

1. Se recomienda modificar la definición de los espectros de diseño del cuerpo principal de las NTCS, con el fin de lograr diseños sísmicos más transparentes, en los que factores relevantes como el factor de sobrerresistencia se empleen de manera explícita, con lo cual sería posible conocer la probable capacidad resistente de la estructura ante sismos, así como valores realistas de sus desplazamientos laterales.

2. En este trabajo se proponen modelos de espectros de diseño inelásticos para ser empleados en los casos de estructuras sobre suelo compresible. En estos espectros se evita el empleo de espectros elásticos, simplificando así los procedimientos de diseño sísmico de estructuras. Además, para obtener estos espectros inelásticos, con base en resultados experimentales observados, se sugiere emplear valores de la fracción de amortiguamiento crítico menores que los comúnmente empleados en la práctica mexicana.

3. Los resultados de este estudio indican que estudios de zonificación sísmica en suelos compresibles en los que sólo se empleen espectros de diseño elásticos serían incompletos, ya que es conveniente especificar espectros de diseño inelásticos sin emplear para su valuación ordenadas espectrales elásticas.

4. Se ha observado el empleo erróneo de espectros de sitio del Apéndice A de las NTCS, por lo que se recomienda especificar restricciones en su empleo que impidan su abuso.

5. Se recomienda que en zonas de alta sismicidad del DF y en el país, no se permita el empleo de estructuras diseñadas con Q igual a 2, es decir se recomienda que en esta zonas sólo se permita el empleo de estructuras diseñadas con Q igual a 4 o en su caso 3.

6. Resultados de ensayes ante cargas laterales de columnas de concreto reforzado indican que sus rigideces estarían sobrestimadas si se calculan con los procedimientos del RCDF, las que en este caso son iguales a las especificadas por el ACI-318. Se recomienda revisar estos procedimientos con el fin de especificar valores realistas de estas rigideces para el análisis estructural. Con este fin, en este trabajo se propone una expresión para valuar el momento de inercia efectivo de columnas rectangulares de concreto reforzado.

7. Se debe mejorar la redacción de la sección 8.4 de las NTCS, incluso cambiar su nombre actual de "Apéndices", con el fin de estipular de manera clara que no sólo los apéndices y otros elementos se deben diseñar para fuerzas sísmicas horizontales, sino también los diafragmas de edificios.

8. Se recomienda mejorar el procedimiento para valuar las fuerzas sísmicas que actúan en apéndices o diafragmas que estipula la sección 8.4 de las NTCS. Para este fin se propone el empleo del procedimiento para valuar las aceleraciones horizontales propuesto por Rodríguez et al. (2002), el cual fue adaptado en este trabajo para ser empleado con los procedimientos de diseño por sismo que estipula las NTCS del DF.

9. Se recomienda la revisión del Apéndice A de las NTCS para evitar incongruencias en el grado de refinamiento que se pretende lograr para valuar diversos parámetros que intervienen en el diseño sísmico.

\section{AGRADECIMIENTOS}

Se agradece la colaboración para obtener algunos resultados de este trabajo de Miguel Torres, Dandy

Roca y Rafael Salinas, del Instituto de Ingeniería, UNAM, y del Dr John J. Blandón, de la Universidad Nacional de Colombia. 


\section{REFERENCIAS}

ACI Committee 318 (ACI 318, 2011), "Building Code Requirements for Reinforced Concrete (ACI 31808)". American Concrete Institute, Farmington Hills, MI.

American Welding Society (AWS, 1998), "Structural Welding Code-Reinforcing Steel” (ANSI/AWS D1.4-98).

American Society for Testing and Materials (2009), "Deformed and Plain Billet-Steel Bars for Concrete Reinforcement” (ASTM A615/A 615M-92b).

Berry M., Parrish M., y Eberhard M. (2004), "Peer Structural Performance Database User's Manual, (Version 1.0)," Pacific Earthquake Engineering Research Center, University of California, Berkeley.

Carr, A. (2002), "RUAUMOKO, Computer Program Library", University of Canterbury, Department of Civil Engineering.

Comisión Federal de Electricidad (1993), "Manual de diseño por Sismo”. México, DF.

Comisión Federal de Electricidad (2008), "Manual de diseño de obras civiles por Sismo”. México, DF.

CSI. (2008), "ETABS Computers and Structures, Inc. User manuals". A Computer Program Library. University Avenue, Berkeley, California 94704, USA.

Dirección General de Normas (2001), "Varilla Corrugada de Acero Proveniente de Lingote y Palanquilla para Refuerzo de Concreto" Norma Mexicana NMX-C-407-ONNCCE-2001

Elwood, K.J., y Eberhard, M.O. (2009), "Effective Stiffness of Reinforced Concrete Columns", ACI Structural Journal, Julio-Agosto, pp 476-484.

Fleischman, R.; Restrepo, J.; Maffei, J. y Seeber, K. "Preview of PCI's New Zealand earthquake reconnaissance team report", Winter 2012, Volume 57, No 1, PCI Journal, pp 42-45.

Gaceta Oficial del Distrito Federal (2004), Reglamento de Construcciones del Distrito Federal. México DF.

Gaceta Oficial del Distrito Federal (2004), "Normas Técnicas Complementarias para Diseño y Construcción de Estructuras de Concreto". Reglamento de Construcciones del Distrito Federal. México DF.

Gaceta Oficial del Distrito Federal (2004), “Normas Técnicas Complementarias para Diseño por Sismo”. Reglamento de Construcciones del Distrito Federal. México DF.

Kam, W. Y., y Pampanin, S. "The seismic performance of RC buildings in the 22 February 2011 Christchurch earthquake", Structural Concrete, Journal of the fib, diciembre 2011, Vol 12, No 4, pp 223-233.

Martinelli, P., y Filippou, F., (2009), "Simulation of the shaking table test of a seven-story shear wall building", Earthquake Engineering-Structural Dynamics, 38, 587-607. 
Meli R, y Avila J. (1989), “The Mexico Earthquake of September 19, 1985-Analysis of Building Response", Earthquake Spectra, Vol 5, No 1, 1-17.

Ordaz, M., Miranda, E., y Avilés, J., (2000), "Propuesta de espectros de diseño por sismo para el D.F.", Memorias VI Simposio Nacional de Ingeniería Sísmica, Querétaro, Qro., México, septiembre, pp. $52-66$.

Panagiotou, M., (2008), "Seismic Design, Testing, and Analysis of Reinforced Concrete Wall Buildings", Tesis Doctoral, University of California, San Diego, USA, supervisada por J. Restrepo.

Park, R., Priestley, M.J., y Gill, W. (1982), "Ductility of Square-Confined Concrete Columns", Journal of the Structural Division, ASCE, V. 108, No ST4, Abril, pp 929-950.

Restrepo J. I. y Rodríguez M. E., (2012a), "On the Probable Moment Strength of Reinforced Concrete Columns". Aceptado para publicación en el ACI Structural Journal, ACI, USA.

Restrepo J. I. y Rodríguez M. E., (2012b), "Yield Displacement and Lateral Stiffness of Reinforced Concrete Columns", trabajo en preparación para ser presentado para posible publicación en el ACI Structural Journal, ACI, USA.

Rodríguez, M., y Botero, J.C. (1995), "Comportamiento sísmico de estructuras considerando propiedades mecánicas de aceros de refuerzo mexicanos". Revista Ingeniería Sísmica, Sociedad Mexicana de Ingeniería Sismica. 1995, No 49, pp 39-50.

Rodriguez M., Blandon J.J. (2005), “Tests on a Half-Scale Two-Story Seismic Resisting Precast Concrete Building", Precast/Prestressed Concrete Institute Journal, Vol. 50, No. 1 (January-February 2005), pp 94-114.

Rodriguez, M., León, G., y Cabrera, H., (2012), "Estudio en mesa vibradora del comportamiento sísmico de un edificio prefabricado de concreto de tres niveles", entregado para su publicación en las Series del Instituto de Ingeniería, Universidad Nacional Autónoma de México.

Rodríguez M. E. y Rodríguez Asabay, J. (2006), "Se debe evitar la soldadura de barras de refuerzo en estructuras de concreto reforzado en zonas sísmicas de México". Revista de Ingeniería Sísmica, Sociedad Mexicana de Ingeniería Sísmica, Vol 75, pp 69-95.

Rodríguez, M., Restrepo, J.I., y Carr, A.J., (2002), "Earthquake induced floor horizontal accelerations in buildings", Earthquake Engineering-Structural Dynamics, 31 693-718.

Rodriguez M. E., Restrepo J. I., y Blandon J. J. (2007), "Seismic Design Forces for Rigid Floor Diaphragms in Precast Concrete Building Structures” Journal of Structural Engineering, ASCE, USA, Noviembre 2007, Vol 133, No 11, pp 1604-1615.

Rodríguez, M., y Torres, M., (2012), "Evaluación del comportamiento sísmico de conexiones trabecolumna de concreto prefabricado con soldadura en las barras de refuerzo. Cambios necesarios para el diseño sísmico de edificios de concreto", enviado para publicación en la Revista de Ingeniería Sísmica, Sociedad Mexicana de Ingeniería Sísmica, México. 
Schoettler M. (2010), "Seismic Demands in Precast Concrete Diaphragms", Tesis para obtener el grado de Doctor of Philosophy in Structural Engineering, University of California, San Diego, USA, supervisada por J. Restrepo.

Uang, C. (1991), "Establishing $R$ (or $R_{w}$ ) and $C_{d}$ Factors for Building Seismic Provisions", Journal of Structural Engineering, ASCE, Vol 117, No 1.

Zermeño, M., Fuentes, A., Aire, C. (1992), "Comportamiento de conexiones entre elementos prefabricados de concreto ante cargas alternadas", Informe Interno No 1704, Instituto de Ingeniería. Universidad Nacional Autónoma de México. 